\title{
Calderón reproducing formulas and applications to Hardy spaces
}

\author{
Pascal Auscher, Alan McIntosh and Andrew J. Morris
}

\begin{abstract}
We establish new Calderón reproducing formulas for selfadjoint operators $D$ that generate strongly continuous groups with finite propagation speed. These formulas allow the analysing function to interact with $D$ through holomorphic functional calculus whilst the synthesising function interacts with $D$ through functional calculus based on the Fourier transform. We apply these to prove the embedding $H_{D}^{p}\left(\wedge T^{*} M\right) \subseteq$ $L^{p}\left(\wedge T^{*} M\right), 1 \leq p \leq 2$, for the Hardy spaces of differential forms introduced by Auscher, McIntosh and Russ, where $D=d+d^{*}$ is the HodgeDirac operator on a complete Riemannian manifold $M$ that has doubling volume growth. This fills a gap in that work. The new reproducing formulas also allow us to obtain an atomic characterisation of $H_{D}^{1}\left(\wedge T^{*} M\right)$. The embedding $H_{L}^{p} \subseteq L^{p}, 1 \leq p \leq 2$, where $L$ is either a divergence form elliptic operator on $\mathbb{R}^{n}$, or a nonnegative self-adjoint operator that satisfies Davies-Gaffney estimates on a doubling metric measure space, is also established in the case when the semigroup generated by the adjoint $-L^{*}$ is ultracontractive.
\end{abstract}

\section{Introduction and main results}

The classical Hardy spaces $H^{p}\left(\mathbb{R}^{n}\right) \subseteq L^{p}\left(\mathbb{R}^{n}\right)$ provide a substitute for the $L^{p}\left(\mathbb{R}^{n}\right)$ scale of spaces on which homogeneous multipliers, such as the Riesz transforms $\left(R_{j} u\right)^{\wedge}(\xi)=i \xi_{j}|\xi|^{-1} \widehat{u}(\xi)$ for $j \in\{1, \ldots, n\}$, are bounded when $p \in[1, \infty)$. It is well known that $H^{p}\left(\mathbb{R}^{n}\right)=L^{p}\left(\mathbb{R}^{n}\right)$ when $p \in(1, \infty)$, whilst $H^{1}\left(\mathbb{R}^{n}\right) \subset L^{1}\left(\mathbb{R}^{n}\right)$, and that $H^{1}\left(\mathbb{R}^{n}\right)$ has an atomic characterisation and a molecular characterisation.

A variety of new Hardy spaces have been designed to obtain a similar theory for useful operators that do not belong to the standard Calderón-Zygmund class. We are primarily motivated by the Hardy spaces of differential forms $H_{D}^{p}\left(\wedge T^{*} M\right)$

Mathematics Subject Classification (2010): Primary 42B30; Secondary 35F35, 35R01, 47B44, 47A60, 58J05.

Keywords: Calderón reproducing formula, Hardy space embedding, self-adjoint operator, finite propagation speed, sectorial operator, off-diagonal estimate, first-order differential operator, Hodge-Dirac operator, divergence form elliptic operator, Riemannian manifold. 
introduced by Auscher, McIntosh and Russ [9]. We temporarily restrict our attention to these spaces, although the main content of the paper contains a more general theory that can be applied to a variety of the contexts considered elsewhere.

The $H_{D}^{p}\left(\wedge T^{*} M\right)$ spaces were designed for the analysis of the Hodge-Dirac operator $D=d+d^{*}$ and the Hodge-Laplacian $\Delta=D^{2}$, where $d$ and $d^{*}$ denote the exterior derivative and its adjoint, acting on the Hilbert space of square integrable differential forms $L^{2}\left(\wedge T^{*} M\right)$ over a complete Riemannian manifold $M$. We will always assume that any such manifold $M$ is smooth and connected, and has doubling volume growth in the sense that there exist constants $A \geq 1$ and $\kappa \geq 0$ such that

$$
0<V(x, \alpha r) \leq A \alpha^{\kappa} V(x, r)<\infty \quad \forall x \in M, \forall r>0, \forall \alpha \geq 1,
$$

where $V(x, r)$ is the Riemannian measure of the geodesic ball $B(x, r)$ in $M$ with centre $x$ and radius $r$. These spaces were designed so that the geometric Riesz transform $D \Delta^{-1 / 2}$ is bounded on $H_{D}^{p}\left(\wedge T^{*} M\right)$ when $p \in[1, \infty]$, and a molecular characterisation was obtained for $H_{D}^{1}\left(\wedge T^{*} M\right)$.

One of the aims of this paper is to show that $H_{D}^{p}\left(\wedge T^{*} M\right) \subseteq L^{p}\left(\wedge T^{*} M\right)$ when $p \in[1,2]$. This result was stated in [9], Corollary 6.3 , but the proof contains a gap that we fill here. Another aim is to show that $H_{D}^{1}\left(\wedge T^{*} M\right)$ has an atomic characterisation, thus strengthening the result in Theorem 6.2 of $[9]$ that $H_{D}^{1}\left(\wedge T^{*} M\right)$ has a molecular characterisation.

We now outline the main ideas. A function $f: S_{\theta}^{o} \rightarrow \mathbb{C}$ is called nondegenerate when it is not identically zero on $\left\{z \in S_{\theta}^{o}: \operatorname{Re} z>0\right\}$ nor on $\left\{z \in S_{\theta}^{o}: \operatorname{Re} z<0\right\}$, where $S_{\theta}^{o}$ is the open bisector in $\mathbb{C}$ of angle $\theta \in(0, \pi / 2)$ defined in (3.1). The space $H_{D}^{p}\left(\wedge T^{*} M\right)$ is defined as a completion of a normed space $E_{D, \psi}^{p}\left(\wedge T^{*} M\right)$ associated with a nondegenerate function $\psi$ from the set

$$
\Psi_{\sigma}^{\tau}\left(S_{\theta}^{o}\right)=\left\{\psi \in H^{\infty}\left(S_{\theta}^{o} \cup\{0\}\right):|\psi(z)| \lesssim \min \left\{|z|^{\sigma},|z|^{-\tau}\right\}\right\},
$$

for some $\sigma, \tau>0$, where $H^{\infty}\left(S_{\theta}^{o} \cup\{0\}\right)$ denotes the algebra of bounded functions on $S_{\theta}^{o} \cup\{0\}$ that are holomorphic on $S_{\theta}^{o}$. We shall not define $E_{D, \psi}^{p}\left(\wedge T^{*} M\right)$ precisely here except to mention that

(1.1) $u \in E_{D, \psi}^{p} \quad$ if and only if $\quad u=\int_{0}^{\infty} \psi_{t}(D) U_{t} \frac{d t}{t} \quad$ for some $U \in T^{p} \cap T^{2}$,

where $T^{p}=T^{p}\left(\left(\wedge T^{*} M\right)_{+}\right)$is an appropriate analogue of the tent space $T^{p}\left(\mathbb{R}_{+}^{n+1}\right)$ introduced by Coifman, Meyer and Stein [15], and $\psi_{t}(D)=\psi(t D)$ is defined by the holomorphic functional calculus of $D$ (see Definition 3.4).

There is an important distinction between a completion of $E_{D, \psi}^{p}$ and the completion of $E_{D, \psi}^{p}$ in $L^{p}$. The former is unique up to isometric isomorphism and can always be constructed as an abstract space, whereas the latter is a unique subspace of $L^{p}$ that may or may not exist. See Section 2 for further details. It was known previously that $E_{D, \psi}^{p} \subseteq L^{p}$ when $\psi$ has suitable decay at the origin and infinity, but this does not guarantee, nor was it proved, that the completion of $E_{D, \psi}^{p}$ in $L^{p}$ exists. Without this property, a completion of $E_{D, \psi}^{p}$ must be interpreted as an 
abstract space consisting of, for example, equivalence classes of Cauchy sequences in $E_{D, \psi}^{p}$ or elements of the second dual space $\left(E_{D, \psi}^{p}\right)^{* *}$. Although various realizations of such an abstract Hardy space were known, these were not shown to be contained in any function space. The approach of Hofmann, Mayboroda and McIntosh in [21], Appendix 2, for instance, can be used to realize the abstract Hardy space as a space of distributions adapted to $D$.

We prove that the completion of $E_{D, \psi}^{p}\left(\wedge T^{*} M\right)$ in $L^{p}\left(\wedge T^{*} M\right)$ exists by utilizing the finite propagation speed of the $C_{0}$-group $\left(e^{i t D}\right)_{t \in \mathbb{R}}$ generated by the HodgeDirac operator $D$ on $L^{2}\left(\wedge T^{*} M\right)$. This provides a constant $c_{D}>0$ such that for all geodesic balls $B(x, r) \subseteq M$, all $u \in L^{2}\left(\wedge T^{*} M\right)$ with $\operatorname{sppt}(u) \subseteq B(x, r)$ and all $t \in \mathbb{R}$, it holds that $\operatorname{sppt}\left(e^{i t D} u\right) \subseteq B\left(x, r+c_{D}|t|\right)$.

The main ideas of the argument are as follows. We use nondegenerate Schwartz functions $\eta$ with compactly supported Fourier transform $\widehat{\eta}$ from the set

$$
\widetilde{\Psi}_{N}^{\delta}(\mathbb{R})=\left\{\eta \in \mathcal{S}(\mathbb{R}): \operatorname{sppt} \widehat{\eta} \subseteq[-\delta, \delta] \text { and } \partial^{k-1} \eta(0)=0 \text { for all } k \in\{1, \ldots, N\}\right\},
$$

for some $\delta>0$ and $N \in \mathbb{N}$, to interact with the finite propagation speed of the group. We will see that for all $t>0$, all $\eta \in \widetilde{\Psi}_{N}^{\delta}(\mathbb{R})$ and all $u \in L^{2}\left(\wedge T^{*} M\right)$ with $\operatorname{sppt}(u) \subseteq B(x, r)$, it holds that $\operatorname{sppt}\left(\eta_{t}(D) u\right) \subseteq B\left(x, r+c_{D} \delta t\right)$, where $\eta_{t}(D)=$ $\eta(t D)$ is defined by the Borel functional calculus of $D$. This is in contrast with a function $\psi \in \Psi_{\sigma}^{\tau}\left(S_{\theta}^{o}\right)$, for which $\psi_{t}(D) u$ may be supported everywhere on $M$.

We incorporate the finite propagation speed into the existing theory by choosing $\psi \in \Psi\left(S_{\theta}^{o}\right)$ and $\eta \in \widetilde{\Psi}(\mathbb{R})$ so that the following Calderón reproducing formula holds:

$$
\int_{0}^{\infty} \psi_{t}(D) \eta_{t}(D) u \frac{d t}{t}=\int_{0}^{\infty} \eta_{t}(D) \psi_{t}(D) u \frac{d t}{t}=u \quad \forall u \in E_{D, \psi}^{p} \cup E_{D, \eta}^{p} .
$$

A comparison of (1.1) and (1.2) shows that if $u \in E_{D, \eta}^{p}$ and $\eta_{t}(D) u \in T^{p} \cap T^{2}$, then $u \in E_{D, \psi}^{p}$. This principle allows us to prove that $E_{D, \psi}^{p}=E_{D, \eta}^{p}$ when the family of operators $\left(\psi_{t}(D) \eta_{s}(D)\right)_{s, t \in(0, \infty)}$ has enough $L^{2}$ off-diagonal decay to control volume growth on the manifold. We then use the Sobolev embedding theorem on geodesic balls and standard energy estimates for the group $\left(e^{i t D}\right)_{t \in \mathbb{R}}$ to prove that the completion of $E_{D, \eta}^{p}$ in $L^{p}$ exists, hence the completion of $E_{D, \psi}^{p}$ in $L^{p}$ exists as well.

Let us remark that the connection between the classical Hardy spaces $H^{p}\left(\mathbb{R}^{n}\right)$ and the tent spaces $T^{p}\left(\mathbb{R}_{+}^{n+1}\right)$ was previously understood in terms of reproducing formulas analogous to (1.2) for convolution operators. In particular, Coifman, Meyer and Stein provided a short proof of the atomic characterisation of $H^{p}\left(\mathbb{R}^{n}\right)$ for $p \in(0,1]$ in $[15]$, Section $9 \mathrm{~b}$, by using the theory of tent spaces and constructing a function $\phi \in C_{c}^{\infty}\left(\mathbb{R}^{n}\right)$ satisfying $\int x^{\gamma} \phi(x) d x=0$ for all $\gamma \in\left[0, N_{p}\right]$ and some $N_{p} \in \mathbb{N}$ depending on $p$ such that

$$
\int_{0}^{\infty} \phi_{(t)} * \partial_{t} P_{(t)} * f d t=f \quad \forall f \in H^{p}\left(\mathbb{R}^{n}\right)
$$

where $P$ is the Poisson kernel and $P_{(t)}(x)=t^{-n} P(x / t)$. This is equivalent to

$$
\int_{0}^{\infty} \widehat{\phi}(t \xi)(-2 \pi t|\xi|) e^{-2 \pi t|\xi|} \frac{d t}{t}=1 \quad \forall \xi \in \mathbb{R}^{n} \backslash\{0\}
$$


from which the analogy with (1.2) is most apparent when $n=1$, since $\eta(x):=\widehat{\phi}(x)$ is in $\widetilde{\Psi}_{N_{p}+1}^{\delta}(\mathbb{R})$ for some $\delta>0$, whilst

$$
\psi(z):= \begin{cases}-2 \pi z e^{-2 \pi z}, & \text { if } \operatorname{Re}(z) \geq 0 \\ 2 \pi z e^{2 \pi z}, & \text { if } \operatorname{Re}(z)<0\end{cases}
$$

is in $\Psi_{1}^{\tau}\left(S_{\theta}^{o}\right)$ for all $\tau>0$ and $\theta \in(0, \pi / 2)$.

After we establish the embedding $H_{D}^{1}\left(\wedge T^{*} M\right) \subseteq L^{1}\left(\wedge T^{*} M\right)$, the finite propagation speed of the group $\left(e^{i t D}\right)_{t \in \mathbb{R}}$ also allows us to obtain an atomic characterisation of $H_{D}^{1}\left(\wedge T^{*} M\right)$. This builds on the molecular characterisation obtained in [9]. The molecular space $H_{D, \operatorname{mol}(N)}^{1}\left(\wedge T^{*} M\right)$ and the atomic space $H_{D \text {,at }(N)}^{1}\left(\wedge T^{*} M\right)$ are introduced in Definition 3.12, where $N \in \mathbb{N}$ is the number of moment conditions satisfied by the molecules and atoms in the respective spaces.

The following theorem summarizes our results for the Hodge-Dirac operator.

Theorem 1.1. Suppose that $M$ is a complete Riemannian manifold satisfying $\left(\mathrm{D}_{\kappa}\right)$ and that $D=d+d^{*}$ is the Hodge-Dirac operator on $L^{2}\left(\wedge T^{*} M\right)$. If $p \in[1,2]$, $\theta \in(0, \pi / 2), \beta>\kappa / 2$ and $\psi \in \Psi_{\beta}\left(S_{\theta}^{o}\right)$ is nondegenerate, then the completion $H_{D, \psi}^{p}\left(\wedge T^{*} M\right)$ of $E_{D, \psi}^{p}\left(\wedge T^{*} M\right)$ in $L^{p}\left(\wedge T^{*} M\right)$ exists. Moreover, if $N \in \mathbb{N}$ and $N>\kappa / 2$, then $H_{D, \psi}^{1}\left(\wedge T^{*} M\right)=H_{D, \operatorname{mol}(N)}^{1}\left(\wedge T^{*} M\right)=H_{D, \text { at }(N)}^{1}\left(\wedge T^{*} M\right)$.

The Hardy space $H_{D, \psi}^{p}\left(\wedge T^{*} M\right)$ in Theorem 1.1 is thus the set of all $u$ in $L^{p}\left(\wedge T^{*} M\right)$ for which there exists a Cauchy sequence $\left(u_{n}\right)_{n}$ in $E_{D, \psi}^{p}\left(\wedge T^{*} M\right)$ that converges to $u$ in $L^{p}\left(\wedge T^{*} M\right)$, together with the norm $\|u\|_{H_{D, \psi}^{p}}=\lim _{n}\left\|u_{n}\right\|_{E_{D, \psi}^{p}}$. The embedding $H_{D, \psi}^{p}\left(\wedge T^{*} M\right) \subseteq L^{p}\left(\wedge T^{*} M\right)$ is then automatic. The comments below Definition 2.1 contain more details.

The results obtained here can also be applied to Hardy spaces designed for higher order operators. In particular, consider the Hardy spaces $H_{L, \psi}^{p}\left(\mathbb{R}^{n}\right)$ introduced by Hofmann, Mayboroda and McIntosh [21] for the analysis of divergence form operators $L=-\operatorname{div} A \nabla=-\sum_{j, k=1}^{n} \partial_{j} A_{j k} \partial_{k}$, acting on $L^{2}\left(\mathbb{R}^{n}\right)$ and interpreted in the usual weak sense via a sesquilinear form, where $A=\left(A_{j k}\right) \in$ $L^{\infty}\left(\mathbb{R}^{n}, \mathcal{L}\left(\mathbb{C}^{n}\right)\right)$ is elliptic in the sense that there exists $\lambda>0$ such that

$$
\operatorname{Re}\langle A(x) \zeta, \zeta\rangle_{\mathbb{C}^{n}} \geq \lambda|\zeta|^{2} \quad \forall \zeta \in \mathbb{C}^{n} \text {, a.e. } x \in \mathbb{R}^{n} .
$$

There exists $\omega_{L} \in[0, \pi / 2)$ such that $L$ is $\omega_{L^{-}}$-sectorial, hence $-L$ and $-L^{*}$ generate analytic semigroups $\left(e^{-t L}\right)_{t>0}$ and $\left(e^{-t L^{*}}\right)_{t>0}$ on $L^{2}\left(\mathbb{R}^{n}\right)$. In order to embed $H_{L, \psi}^{p}\left(\mathbb{R}^{n}\right)$ in $L^{p}\left(\mathbb{R}^{n}\right)$ when $1 \leq p \leq 2$, we assume that there exists $g \in L_{\text {loc }}^{2}((0, \infty))$ such that

$$
\left\|e^{-t L^{*}} u\right\|_{\infty} \leq g(t)\|u\|_{2} \quad \forall u \in L^{2}\left(\mathbb{R}^{n}\right), \forall t>0 .
$$

Let us remark that (1.4) is equivalent to the action of the semigroup $\left(e^{-t L}\right)_{t>0}$ from $L^{1}\left(\mathbb{R}^{n}\right)$ to $L^{2}\left(\mathbb{R}^{n}\right)$ (it is usually called ultracontractivity). Hence, this action of the semigroup on $L^{1}\left(\mathbb{R}^{n}\right)$ suffices to obtain $H_{L, \psi}^{1}\left(\mathbb{R}^{n}\right)$ as a subspace of $L^{1}\left(\mathbb{R}^{n}\right)$ in Theorem 1.2 below. 
Let us also remark that $(1.4)$ is immediate when the semigroup $\left(e^{-t L^{*}}\right)_{t>0}$ has a kernel $\left(K_{t}(\cdot, \cdot)\right)_{t>0}$ defined pointwise almost everywhere on $\mathbb{R}^{n} \times \mathbb{R}^{n}$ with the property that for each $T>0$, there exist constants $C_{T}, c_{T}>0$ such that

$$
\left|K_{t}(x, y)\right| \leq C_{T} t^{-n / 2} e^{-c_{T}|x-y|^{2} / t} \quad \forall x, y \in \mathbb{R}^{n}, \forall t \in(0, T] .
$$

In fact, property (1.4) is usually obtained as a step toward proving (1.5). For example, the local Gaussian estimates in (1.5) hold when, in addition to having $A$ bounded and elliptic, $A$ is uniformly continuous (see Theorem 4.8 in [4]) or belongs to VMO or has small BMO norm (see Chapter 1 in [10]).

The following theorem is essentially known when (1.5) holds (see the remark below Proposition 9.1 in [21]). We provide a short proof when (1.4) holds as an application of our techniques.

Theorem 1.2. Suppose that $A \in L^{\infty}\left(\mathbb{R}^{n}, \mathcal{L}\left(\mathbb{C}^{n}\right)\right)$ is elliptic and that $L=-\operatorname{div} A \nabla$ on $L^{2}\left(\mathbb{R}^{n}\right)$ satisfies (1.4). If $p \in[1,2], \theta \in\left(\omega_{L}, \pi / 2\right), \beta>n / 4$ and $\psi \in \Psi_{\beta}\left(S_{\theta}^{o}\right)$ is nondegenerate, then the completion $H_{L, \psi}^{p}\left(\mathbb{R}^{n}\right)$ of $E_{L, \psi}^{p}\left(\mathbb{R}^{n}\right)$ in $L^{p}\left(\mathbb{R}^{n}\right)$ exists. Moreover, if $N \in \mathbb{N}$ and $N>n / 4$, then $H_{L, \psi}^{1}\left(\mathbb{R}^{n}\right)=H_{L, \operatorname{mol}(N)}^{1}\left(\mathbb{R}^{n}\right)$, and when $A$ is self-adjoint, then also $H_{L, \psi}^{1}\left(\mathbb{R}^{n}\right)=H_{L, \text { at }(N)}^{1}\left(\mathbb{R}^{n}\right)$.

A theory of Hardy spaces was developed by Hofmann, Lu, Mitrea, Mitrea and Yan [20] for nonnegative self-adjoint operators $L$ satisfying Davies-Gaffney estimates (see (5.1)) on doubling metric measure spaces $M$. For example, when $A$ is self-adjoint, then $L=-\operatorname{div} A \nabla$ has these properties. The framework developed here provides an embedding for these spaces when $L$ acts on a vector bundle $\mathcal{V}$ over $M$, as defined in Section 2, and there exists $g \in L_{\text {loc }}^{2}((0, \infty))$ such that

$$
\left\|e^{-t L} u\right\|_{\infty} \leq g(t)\|u\|_{2} \quad \forall u \in L^{2}(\mathcal{V}), \forall t>0 .
$$

In this context, since $L$ is self-adjoint, it is well known that (1.6) is equivalent to pointwise kernel estimates for the semigroup $\left(e^{-t L}\right)_{t>0}$ (see Lemma 2.1.2 in [18]).

Theorem 1.3. Suppose that $M$ is a doubling metric measure space satisfying $\left(\mathrm{D}_{\kappa}\right)$ and that $L$ is a nonnegative self-adjoint operator on $L^{2}(\mathcal{V})$ satisfying DaviesGaffney estimates and (1.6). If $p \in[1,2], \theta \in(0, \pi / 2), \beta>\kappa / 4$ and $\psi \in \Psi_{\beta}\left(S_{\theta}^{o}\right)$ is nondegenerate, then the completion $H_{L, \psi}^{p}(\mathcal{V})$ of $E_{L, \psi}^{p}(\mathcal{V})$ in $L^{p}(\mathcal{V})$ exists. Moreover, if $N \in \mathbb{N}$ and $N>\kappa / 4$, then $H_{L, \psi}^{1}(\mathcal{V})=H_{L, \operatorname{mol}(N)}^{1}(\mathcal{V})=H_{L, \operatorname{at}(N)}^{1}(\mathcal{V})$.

It remains an open question as to whether Theorems 1.2 and 1.3 hold in the absence of ultracontractivity estimates such as (1.4) and (1.6). The first-order methods developed here, however, provide a new proof of Theorem 1.2 that does not rely on ultracontractivity but instead requires that $A$ is self-adjoint with smooth coefficients. We present this proof at the conclusion of the paper as a basis for future work.

The structure of the paper is as follows. In Section 2, we fix notation and discuss when the completion of a normed space inside a given Banach space exists. In Section 3, we briefly recast the theory of Hardy spaces from [9] in the context 
of a vector bundle $\mathcal{V}$ over a doubling metric measure space $M$ for any operator $\mathcal{D}$ on $L^{2}(\mathcal{V})$ that is bisectorial with a bounded holomorphic functional calculus and that satisfies polynomial off-diagonal estimates. We then introduce an additional hypothesis $(\mathrm{H} 4)_{\Psi}$ on $\mathcal{D}$, based on the $\Psi\left(S_{\theta}^{o}\right)$ class, that guarantees the embedding $H_{\mathcal{D}}^{p}(\mathcal{V}) \subseteq L^{p}(\mathcal{V})$, when $p \in[1,2]$, and the molecular characterisation of $H_{\mathcal{D}}^{1}(\mathcal{V})$. This is the content of Theorems 3.10 and 3.13.

In Section 4, we restrict consideration to any operator $D$ that is self-adjoint on $L^{2}(\mathcal{V})$ and for which the associated $C_{0}$-group $\left(e^{i t D}\right)_{t \in \mathbb{R}}$ has finite propagation speed. This allows us to introduce an alternative hypothesis $(\mathrm{H} 4)_{\widetilde{\Psi}}$ on $D$, based on the $\widetilde{\Psi}(\mathbb{R})$ class, that guarantees the embedding $H_{D}^{p}(\mathcal{V}) \subseteq L^{p}(\mathcal{V})$, when $p \in[1,2]$, and the atomic characterisation of $H_{D}^{1}(\mathcal{V})$. This is the content of Theorems 4.7 and 4.9. In Theorem 4.11, we verify $(\mathrm{H} 4)_{\widetilde{\Psi}}$ when $M$ is a complete Riemannian manifold and $D$ is a smooth-coefficient, self-adjoint, first-order, differential operator with bounded principal symbol

The results for the Hodge-Dirac operator $D=d+d^{*}$ and the divergence form operator $L=-\operatorname{div} A \nabla$ in Theorems 1.1 and 1.2 are deduced in Sections 3.2 and 4.2. In Section 5, we combine the techniques of the preceding two sections to prove Theorem 1.3. Section 6 is an appendix that contains the technical offdiagonal estimates used to prove Theorems 4.7 and 4.9 .

\section{Notation and preliminaries}

Throughout the paper, let $M$ denote a metric measure space with a metric $\rho$ and a $\sigma$-finite measure $\mu$ that is Borel with respect to the $\rho$-topology. A ball in $M$ will always refer to an open $\rho$-ball. For $x \in M$ and $\alpha, r>0$, let $B(x, r)$ denote the ball in $M$ with centre $x$ and radius $r$, let $V(x, r)=\mu(B(x, r))$ and $(\alpha B)(x, r)=B(x, \alpha r)$. The metric measure space $M$ is called doubling when there exist constants $A \geq 1$ and $\kappa \geq 0$ such that

$$
0<V(x, \alpha r) \leq A \alpha^{\kappa} V(x, r)<\infty \quad \forall x \in M, \forall r>0, \forall \alpha \geq 1
$$

For any $E, F \subseteq M$, set $\rho(E, F)=\inf \{\rho(x, y): x \in E, y \in F\}$.

A vector bundle $\mathcal{V}$ over $M$ refers to a complex vector bundle $\pi: \mathcal{V} \rightarrow M$ equipped with a Hermitian metric $\langle\cdot, \cdot\rangle_{x}$ that depends continuously on $x \in M$. For any vector bundle $\mathcal{V}$, there are naturally defined Banach spaces $L^{p}(\mathcal{V}), 1 \leq$ $p \leq \infty$, of measurable sections. The Hilbert space $L^{2}(\mathcal{V})$ of square integrable sections of $\mathcal{V}$ has the inner product $\langle u, v\rangle=\int_{M}\langle u(x), v(x)\rangle_{x} d \mu(x)$. For any linear operator $T$ on $L^{2}(\mathcal{V})$, the domain $\operatorname{Dom}(T)$, range $\mathrm{R}(T)$ and null space $\mathrm{N}(T)$ are subspaces of $L^{2}(\mathcal{V})$, and the operator norm $\|T\|=\sup \left\{\|T u\|_{L^{2}(\mathcal{V})} /\|u\|_{L^{2}(\mathcal{V})}: u \in\right.$ $\operatorname{Dom}(T), u \neq 0\}$. The Banach algebra of all bounded linear operators on $L^{2}(\mathcal{V})$ is denoted by $\mathcal{L}\left(L^{2}(\mathcal{V})\right)$.

For normed spaces $X$ and $Y$, we write $X \subseteq Y$ when $X$ is a subset of $Y$ with the property that there exists $C>0$ such that $\|x\|_{Y} \leq C\|x\|_{X}$ for all $x \in X$, and we write $X=Y$ when $X \subseteq Y \subseteq X$. A completion $(\mathcal{X}, \imath)$ of a normed space $X$ consists of a Banach space $\mathcal{X}$ and an isometry $\imath: X \rightarrow \mathcal{X}$ such that $\imath(X)$ is dense 
in $\mathcal{X}$. Every normed space has a completion but this abstract construction is not sufficient for our purposes. It is convenient to formalise the following related notion.

Definition 2.1. Let $X$ be a normed space and suppose that $X \subseteq Y$ for some Banach space $Y$. A Banach space $\widetilde{X}$ is called the completion of $X$ in $Y$ when $X \subseteq \widetilde{X} \subseteq Y$, the set $X$ is dense in $\widetilde{X}$, and $\|x\|_{X}=\|x\|_{\widetilde{X}}$ for all $x \in X$.

It is easily checked that the completion $\widetilde{X}$ of $X$ in $Y$ is unique whenever it exists. Moreover, the set $\widetilde{X}$ consists of all $x$ in $Y$ for which there is a Cauchy sequence $\left(x_{n}\right)_{n}$ in $X$ such that $\left(x_{n}\right)_{n}$ converges to $x$ in $Y$, and with the norm $\|x\|_{\tilde{X}}=$ $\lim _{n \rightarrow \infty}\left\|x_{n}\right\|_{X}$, the space $\left(\tilde{X},\|\cdot\|_{\tilde{X}}\right)$ is complete. This can be deduced from the following necessary and sufficient conditions for the existence of a completion inside a given Banach space. The proof is left to the reader.

Proposition 2.2. Let $X$ be a normed space and suppose that $X \subseteq Y$ for some Banach space $Y$, so the identity $I: X \rightarrow Y$ is bounded. The following are equivalent:

(1) the completion of $X$ in $Y$ exists;

(2) if $(\mathcal{X}, \imath)$ is a completion of $X$, then the unique operator $\widetilde{I}$ in $\mathcal{L}(\mathcal{X}, Y)$ defined by the commutative diagram below,

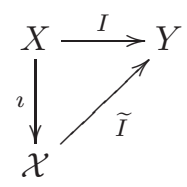

is injective;

(3) for each Cauchy sequence $\left(x_{n}\right)_{n}$ in $X$ that converges to 0 in $Y$, it follows that $\left(x_{n}\right)_{n}$ converges to 0 in $X$.

We adopt the convention for estimating $x, y \geq 0$ whereby $x \lesssim y$ means that there exists a constant $C \geq 1$, which only depends on constants specified in the relevant preceding hypotheses, such that $x \leq C y$. We write $x \approx y$ when $x \lesssim y \lesssim x$. The set of positive integers is denoted by $\mathbb{N}$ whilst $\mathbb{N}_{0}=\mathbb{N} \cup\{0\}$ and $\mathbb{R}_{+}=(0, \infty)$. Finally, we apologise in advance for the excess of notation, but it is required to handle some delicate points.

\section{Sectorial operators with off-diagonal estimates}

Auscher, McIntosh and Russ [9] designed the Hardy spaces of differential forms $H_{D}^{p}\left(\wedge T^{*} M\right), 1 \leq p \leq \infty$, for the Hodge-Dirac operator $D=d+d^{*}$ acting on $L^{2}\left(\wedge T^{*} M\right)$ over a doubling Riemannian manifold $M$. We briefly recast that theory in the context of a vector bundle $\mathcal{V}$ over a doubling metric measure space $(M, \rho, \mu)$. Instead of the Hodge-Dirac operator, we consider any closed, densely 
defined operator $\mathcal{D}: \operatorname{Dom}(\mathcal{D}) \subseteq L^{2}(\mathcal{V}) \rightarrow L^{2}(\mathcal{V})$ that is bisectorial with a bounded holomorphic functional calculus (e.g. this holds when $\mathcal{D}$ is self-adjoint) and satisfies polynomial off-diagonal estimates (e.g. these hold for suitable classes of differential operators $\mathcal{D}$, not necessarily of first-order). The setup below allows us to define these properties.

For $0 \leq \mu<\theta<\pi / 2$, define the following bisectors in the complex plane:

$$
\begin{aligned}
& S_{\mu}=\{z \in \mathbb{C}: z=0 \text { or }|\arg z| \leq \mu \text { or }|\pi-\arg z| \leq \mu\} ; \\
& S_{\theta}^{o}=\{z \in \mathbb{C} \backslash\{0\}:|\arg z|<\theta \text { or }|\pi-\arg z|<\theta\} .
\end{aligned}
$$

A function on $S_{\theta}^{o}$ is called nondegenerate when it is not identically zero on each component of $S_{\theta}^{o}$. The algebra of bounded complex-valued functions on $S_{\theta}^{o} \cup\{0\}$ that are holomorphic on $S_{\theta}^{o}$ is denoted by $H^{\infty}\left(S_{\theta}^{o} \cup\{0\}\right)$. For $\sigma, \tau>0$, define

$$
\Psi_{\sigma}^{\tau}\left(S_{\theta}^{o}\right)=\left\{\psi \in H^{\infty}\left(S_{\theta}^{o} \cup\{0\}\right):|\psi(z)| \lesssim \min \left\{|z|^{\sigma},|z|^{-\tau}\right\}\right\},
$$

$\Psi_{\sigma}\left(S_{\theta}^{o}\right)=\bigcup_{\tau>0} \Psi_{\sigma}^{\tau}\left(S_{\theta}^{o}\right), \Psi^{\tau}\left(S_{\theta}^{o}\right)=\bigcup_{\sigma>0} \Psi_{\sigma}^{\tau}\left(S_{\theta}^{o}\right)$ and $\Psi\left(S_{\theta}^{o}\right)=\bigcup_{\sigma>0} \bigcup_{\tau>0} \Psi_{\sigma}^{\tau}\left(S_{\theta}^{o}\right)$. For functions $f: S_{\theta}^{o} \rightarrow \mathbb{C}$, define $f^{*}(z)=\overline{f(\bar{z})}$, and for $t>0$, define $f_{t}(z)=f(t z)$.

Consider the following hypotheses concerning a closed, densely defined operator $\mathcal{D}: \operatorname{Dom}(\mathcal{D}) \subseteq L^{2}(\mathcal{V}) \rightarrow L^{2}(\mathcal{V})$, where $\mathbf{1}_{E}$ denotes the characteristic function of a measurable set $E \subseteq M$, and $\langle\alpha\rangle=\min \{\alpha, 1\}$ and $\langle\alpha / 0\rangle=1$ when $\alpha>0$.

(H1) There exists $\omega \in[0, \pi / 2)$ such that $\mathcal{D}$ is type $S_{\omega}$, which is defined to mean that the spectrum $\sigma(\mathcal{D}) \subseteq S_{\omega}$ and that for each $\theta \in(\omega, \pi / 2)$, there exists $C_{\theta}>0$ such that $\left\|(z I-\mathcal{D})^{-1} u\right\|_{2} \leq C_{\theta}\|u\|_{2} /|z|$ for all $z \in \mathbb{C} \backslash S_{\theta}$ and $u \in L^{2}(\mathcal{V})$.

(H2) For each $\theta \in(\omega, \pi / 2)$, the operator $\mathcal{D}$ has a bounded $H^{\infty}\left(S_{\theta}^{o} \cup\{0\}\right)$ functional calculus in $L^{2}(\mathcal{V})$, which is defined to mean that there exists $c_{\theta}>0$ such that $\|\psi(\mathcal{D}) u\|_{2} \leq c_{\theta}\|\psi\|_{\infty}\|u\|_{2}$ for all $\psi \in \Psi\left(S_{\theta}^{o}\right)$ and $u \in L^{2}(\mathcal{V})$.

(H3) There exists $m \in \mathbb{N}$ such that for each $\theta \in(\omega, \pi / 2)$ and $N \in \mathbb{N}$ it holds that

$$
\left\|\mathbf{1}_{E}(z I-\mathcal{D})^{-1} \mathbf{1}_{F} u\right\|_{2} \leq \frac{C_{\theta, N}}{|z|}\left\langle\frac{1}{\rho(E, F)^{m}|z|}\right\rangle^{N}\|u\|_{2}
$$

for all $z \in \mathbb{C} \backslash S_{\theta}, u \in L^{2}(\mathcal{V})$, measurable sets $E, F \subseteq M$, and some $C_{\theta, N}>0$.

Let us note that (H1) is implicit in (H2) and (H3). It is well known that (H1) and (H2) hold with $\omega=0, C_{\theta}=1 / \sin \theta$ and $c_{\theta}=1$, whenever $\mathcal{D}$ is self-adjoint. The number $m$ in (H3) indicates that the off-diagonal estimates associated with $\mathcal{D}$ resemble those associated with an $m$ th-order differential operator.

The theory of type $S_{\omega}$ operators is well known (see, for instance, [26], [1], [8]). If (H1) holds, then for $\theta \in(\omega, \pi / 2)$ and $\psi \in \Psi\left(S_{\theta}^{o}\right)$, define $\psi(\mathcal{D}) \in \mathcal{L}\left(L^{2}(\mathcal{V})\right)$ by

$$
\psi(\mathcal{D}) u=\frac{1}{2 \pi i} \int_{\partial S_{\mu}^{o}} \psi(z)(z I-\mathcal{D})^{-1} u d z \quad \forall u \in L^{2}(\mathcal{V}),
$$


where $\mu \in(\omega, \theta)$ is arbitrary and $\partial S_{\mu}^{o}$ is the positively oriented boundary of $S_{\mu}^{o}$. It holds that $L^{2}(\mathcal{V})=\overline{\mathrm{R}(\mathcal{D})} \oplus N(\mathcal{D})$ when $\mathcal{D}$ is type $S_{\omega}$ (see Theorem 3.8 in [16]) and so

$$
\psi(\mathcal{D}) u=\mathrm{P}_{\overline{\mathrm{R}(\mathcal{D})}} \psi(\mathcal{D}) \mathrm{P}_{\overline{\mathrm{R}(\mathcal{D})}} u \quad \forall u \in L^{2}(\mathcal{V}),
$$

where $\mathrm{P}_{\overline{\mathrm{R}(\mathcal{D})}}$ denotes the projection from $L^{2}(\mathcal{V})$ onto $\overline{\mathrm{R}(\mathcal{D})}$ (see Lemma 4.5 in [28]).

It is well known (see [1], [26]) that (H2) holds if and only if the quadratic estimate

$$
\int_{0}^{\infty}\left\|\psi_{t}(\mathcal{D}) u\right\|_{2}^{2} \frac{d t}{t} \approx\|u\|^{2} \quad \forall u \in \overline{\mathrm{R}(\mathcal{D})}
$$

holds for all nondegenerate $\psi \in \Psi\left(S_{\theta}^{o}\right)$, where $\psi_{t}(z)=\psi(t z)$. If (H2) holds, then for $f \in H^{\infty}\left(S_{\theta}^{o} \cup\{0\}\right)$, define $f(\mathcal{D}) \in \mathcal{L}\left(L^{2}(\mathcal{V})\right)$ satisfying $\|f(\mathcal{D})\| \leq c_{\theta}\|f\|_{\infty}$ by

$$
f(\mathcal{D}) u=\lim _{n \rightarrow \infty}\left(f \psi_{(n)}\right)(\mathcal{D}) u+f(0) \mathrm{P}_{N(\mathcal{D})} u \quad \forall u \in L^{2}(\mathcal{V})
$$

where $\left(\psi_{(n)}\right)_{n \in \mathbb{N}}$ is an arbitrary sequence of uniformly bounded functions in $\Psi\left(S_{\theta}^{o}\right)$ that converges to 1 uniformly on compact subsets of $S_{\theta}^{o}$. The mapping $f \mapsto f(\mathcal{D})$ given by (3.5) is the unique algebra homomorphism from $H^{\infty}\left(S_{\theta}^{o} \cup\{0\}\right)$ into $\mathcal{L}\left(L^{2}(\mathcal{V})\right)$ with the following properties (see Lecture 2 in [1]):

(3.6) If $\mathbf{1}(z)=1$ on $S_{\theta}^{o} \cup\{0\}$, then $\mathbf{1}(\mathcal{D})=I$ on $L^{2}(\mathcal{V})$;

(3.7) If $\lambda \in \mathbb{C} \backslash S_{\omega}$ and $f(z)=(\lambda-z)^{-1}$ on $S_{\theta}^{o} \cup\{0\}$, then $f(\mathcal{D})=(\lambda I-\mathcal{D})^{-1}$;

(3.8) If $\left(f_{n}\right)_{n}$ is a sequence in $H^{\infty}\left(S_{\theta}^{o} \cup\{0\}\right)$ that converges uniformly on compact sets to a function $f$ in $H^{\infty}\left(S_{\theta}^{O} \cup\{0\}\right)$, and $\sup _{n}\left\|f_{n}\right\|_{\infty}<\infty$, then $\lim _{n} f_{n}(\mathcal{D}) u=f(\mathcal{D}) u$ for all $u \in L^{2}(\mathcal{V})$.

Hypotheses (H1)-(H3) are sufficient to construct Hardy spaces $H_{\mathcal{D}}^{p}(\mathcal{V})$ as in $[9]$. To begin, we use (3.2) to obtain the following extension of Lemma 3.6 in [9] (for the improved $\Psi\left(S_{\theta}^{o}\right)$ class exponents presented here, see Lemma 7.3 in [23]): If $0<\delta<\sigma, \theta \in(\omega, \pi / 2)$ and $\psi \in \Psi_{\sigma}\left(S_{\theta}^{o}\right)$, then there exists $C>0$ such that

$$
\left\|\mathbf{1}_{E}\left(f \psi_{t}\right)(\mathcal{D}) \mathbf{1}_{F} u\right\|_{2} \leq C\|f\|_{\infty}\left\langle\frac{t}{\rho(E, F)^{m}}\right\rangle^{\sigma-\delta}\|u\|_{2}
$$

for all $t>0, f \in H^{\infty}\left(S_{\theta}^{o} \cup\{0\}\right), u \in L^{2}(\mathcal{V})$, and measurable sets $E, F \subseteq M$.

The theory of tent spaces $T^{p}\left(\mathbb{R}_{+}^{n+1}\right)$ developed by Coifman, Meyer and Stein [15] has the following extension when $\pi: \mathcal{V} \rightarrow M$ is a vector bundle over a doubling metric measure space $M$. Let $\mathcal{V}_{+}$denote the vector bundle $\pi_{+}: \mathcal{V} \times \mathbb{R}_{+} \rightarrow M \times \mathbb{R}_{+}$ over $M \times \mathbb{R}_{+}$defined by $\pi_{+}(v, t):=(\pi(v), t)$ for all $v \in \mathcal{V}, t \in \mathbb{R}_{+}$. For $x \in M$, $t \in \mathbb{R}_{+}$and sections $U, V$ of $\mathcal{V}_{+}$, since $U(x, t) \in \pi^{-1}(\{x\}) \times\{t\}$, we let $U_{t}(x)$ denote the component of $U(x, t)$ in $\pi^{-1}(\{x\})$, and define the Hermitian metric on $\mathcal{V}_{+}$by $\langle U(x, t), V(x, t)\rangle_{x, t}:=\left\langle U_{t}(x), V_{t}(x)\right\rangle_{x}$. For $p \in[1, \infty)$, the tent space $T^{p}\left(\mathcal{V}_{+}\right)$is the Banach space of all $U$ in $L_{\text {loc }}^{2}\left(\mathcal{V}_{+}\right)$satisfying

$$
\|U\|_{T^{p}}:=\left(\int_{M}\left(\iint_{\Gamma(x)}\left|U_{t}(y)\right|_{y}^{2} \frac{d \mu(y)}{V(y, t)} \frac{d t}{t}\right)^{p / 2} d \mu(x)\right)^{1 / p}<\infty,
$$


where the cone $\Gamma(x)=\left\{(y, t) \in M \times \mathbb{R}_{+} \mid \rho(x, y)<t\right\}$. The tent space $T^{\infty}\left(\mathcal{V}_{+}\right)$is the Banach space of all $U$ in $L_{\text {loc }}^{2}\left(\mathcal{V}_{+}\right)$satisfying

$$
\|U\|_{T^{\infty}}:=\sup _{x \in M} \sup _{B \in \mathcal{B}(x)}\left(\frac{1}{\mu(B)} \iint_{T(B)}\left|U_{t}(y)\right|_{y}^{2} d \mu(y) \frac{d t}{t}\right)^{1 / 2}<\infty,
$$

where $\mathcal{B}(x)$ denotes the set of all balls $B \subseteq M$ with the property that $x \in B$, and the tent $T(B)=\left\{(y, t) \in M \times \mathbb{R}_{+} \mid \rho(y, M \backslash B) \geq t\right\}$.

We require the following properties, which can be proved as in the references cited when $M$ is a doubling metric measure space:

(3.10) If $p \in[1, \infty)$ and $1 / p+1 / p^{\prime}=1$, then $T^{p^{\prime}}$ is realized as the dual of $T^{p}$ by the pairing $\langle U, V\rangle_{T^{2}}:=\int_{0}^{\infty} \int_{M}\left\langle U_{t}(x), V_{t}(x)\right\rangle_{x} d \mu(x) d t / t$ (see [15]);

(3.11) If $\theta \in(0,1), 1 \leq p_{0}<p_{1} \leq \infty$ and $1 / p_{\theta}=(1-\theta) / p_{0}+\theta / p_{1}$, then the complex interpolation space $\left[T^{p_{0}}, T^{p_{1}}\right]_{\theta}=T^{p_{\theta}}$ (see [22], [11], [14], [2]).

There is also the following atomic characterisation of $T^{1}\left(\mathcal{V}_{+}\right)$, for which a section $A \in L^{2}\left(\mathcal{V}_{+}\right)$is called a $T^{1}$-atom when there is a ball $B \subseteq M$ such that $A$ is supported on the tent $T(B)$ and the norm $\|A\|_{T^{2}} \leq \mu(B)^{-1 / 2}$.

Theorem 3.1. Suppose that $\mathcal{V}$ is a vector bundle over a doubling metric measure space $M$ and that $p \in[1, \infty)$. For each $U$ in $T^{1}\left(\mathcal{V}_{+}\right) \cap T^{p}\left(\mathcal{V}_{+}\right)$, there exist a sequence $\left(\lambda_{j}\right)_{j}$ in $\ell^{1}$ and a sequence $\left(A_{j}\right)_{j}$ of $T^{1}$-atoms such that $\sum_{j} \lambda_{j} A_{j}$ converges to $U$ in $T^{1}\left(\mathcal{V}_{+}\right)$, in $T^{p}\left(\mathcal{V}_{+}\right)$and almost everywhere in $M \times \mathbb{R}_{+}$, such that $\|U\|_{T^{1}} \approx\left\|\left(\lambda_{j}\right)_{j}\right\|_{\ell^{1}}$.

Proof. This follows the proof of Theorem 1.1 in [29], which is based on Theorem 1 in [15]. The convergence in $T^{p}$ is not explicit in those references, but it follows by dominated convergence, as in [21], Proposition 3.25, or [12], Theorem 3.6.

We follow [9] to begin the development of Hardy spaces $H_{\mathcal{D}}^{p}(\mathcal{V})$ in earnest.

Definition 3.2. Suppose that $\mathcal{D}$ satisfies $(\mathrm{H} 1)-(\mathrm{H} 3)$ on $L^{2}(\mathcal{V})$ for some $\omega \in[0, \pi / 2)$ and $m \in \mathbb{N}$. For $\theta \in(\omega, \pi / 2)$ and $\psi \in \Psi\left(S_{\theta}^{o}\right)$, define $\mathcal{Q}_{\psi}^{\mathcal{D}}$ in $\mathcal{L}\left(L^{2}, T^{2}\right)$ by

$$
\left(\mathcal{Q}_{\psi}^{\mathcal{D}} u\right)_{t}=\psi\left(t^{m} \mathcal{D}\right) u \quad \forall t>0, \forall u \in L^{2}(\mathcal{V})
$$

and $\mathcal{S}_{\psi}^{\mathcal{D}}$ in $\mathcal{L}\left(T^{2}, L^{2}\right)$ by

$$
\mathcal{S}_{\psi}^{\mathcal{D}} U=\int_{0}^{\infty} \psi\left(s^{m} \mathcal{D}\right) U_{s} \frac{d s}{s} \quad \forall U \in T^{2}\left(\mathcal{V}_{+}\right) .
$$

The operator $\mathcal{Q}_{\psi}^{\mathcal{D}}$ is bounded because (H2) is equivalent to the quadratic estimate in (3.4). The operator $\mathcal{S}_{\psi}^{\mathcal{D}}$ is bounded because $\mathcal{S}_{\psi}^{\mathcal{D}}=\left(\mathcal{Q}_{\psi^{*}}^{\mathcal{D}^{*}}\right)^{*}$ and the adjoint $\mathcal{D}^{*}$ satisfies (H2) if and only if $\mathcal{D}$ satisfies (H2) (see, for instance, Lecture 3 in [1]). These operators provide the following Calderón reproducing formula (see Remark 2.1 in [9]). 
Proposition 3.3. Suppose that $\mathcal{D}$ satisfies $(\mathrm{H} 1)-(\mathrm{H} 3)$ for some $\omega \in[0, \pi / 2)$ and $m \in \mathbb{N}$. If $\sigma, \tau>0, \theta \in(\omega, \pi / 2)$ and $\psi \in \Psi\left(S_{\theta}^{o}\right)$ is nondegenerate, then there exists a nondegenerate $\tilde{\psi} \in \Psi_{\sigma}^{\tau}\left(S_{\theta}^{o}\right)$ such that $\mathcal{S}_{\psi}^{\mathcal{D}} \mathcal{Q}_{\tilde{\psi}}^{\mathcal{D}} u=\mathcal{S}_{\tilde{\psi}}^{\mathcal{D}} \mathcal{Q}_{\psi}^{\mathcal{D}} u=\mathrm{P}_{\overline{R(\mathcal{D})}} u$ for all $u \in L^{2}(\mathcal{V})$.

In preparation for defining the Hardy space $H_{\mathcal{D}, \psi}^{p}(\mathcal{V})$, we now define a possibly incomplete space $E_{\mathcal{D}, \psi}^{p}(\mathcal{V})$.

Definition 3.4. Suppose that $\mathcal{D}$ satisfies $(\mathrm{H} 1)-(\mathrm{H} 3)$ on $L^{2}(\mathcal{V})$ for some $\omega \in[0, \pi / 2)$ and $m \in \mathbb{N}$. For $\theta \in(\omega, \pi / 2), \psi \in \Psi\left(S_{\theta}^{o}\right)$ and $p \in[1, \infty]$, the space $E_{\mathcal{D}, \psi}^{p}(\mathcal{V})$ consists of the set $\mathcal{S}_{\psi}^{\mathcal{D}}\left(T^{p} \cap T^{2}\right)$ together with the seminorm

$$
\|u\|_{E_{\mathcal{D}, \psi}^{p}}:=\inf \left\{\|U\|_{T^{p}}: U \in T^{p} \cap T^{2} \text { and } u=\mathcal{S}_{\psi}^{\mathcal{D}} U\right\}
$$

for all $u \in \mathcal{S}_{\psi}^{\mathcal{D}}\left(T^{p} \cap T^{2}\right)$.

In [9], the Hardy space $H_{\mathcal{D}, \psi}^{p}(\mathcal{V})$ is defined to be an abstract completion of $E_{\mathcal{D}, \psi}^{p}(\mathcal{V})$. Our question here is whether we can define $H_{\mathcal{D}, \psi}^{p}(\mathcal{V})$ to be the completion of $E_{\mathcal{D}, \psi}^{p}(\mathcal{V})$ in $L^{p}(\mathcal{V})$. So does the completion of $E_{\mathcal{D}, \psi}^{p}(\mathcal{V})$ in $L^{p}(\mathcal{V})$ exist? This is immediate when (H2) holds and $p=2$, since for each $\theta \in(\omega, \pi / 2)$ and nondegenerate $\psi \in \Psi\left(S_{\theta}^{o}\right)$, we have by $(3.3)$, (3.4) and Proposition 3.3 that $\mathcal{S}_{\psi}^{\mathcal{D}}\left(T^{2}\right)=\overline{\mathrm{R}(\mathcal{D})}$ with

$$
\|u\|_{E_{\mathcal{D}, \psi}^{2}} \approx\left\|\mathcal{Q}_{\psi}^{\mathcal{D}} u\right\|_{T^{2}} \approx\|u\|_{2} \quad \forall u \in \overline{\mathrm{R}(\mathcal{D})} .
$$

This motivates the following definition.

Definition 3.5. Suppose that $\mathcal{D}$ satisfies $(\mathrm{H} 1)-(\mathrm{H} 3)$ on $L^{2}(\mathcal{V})$ for some $\omega \in[0, \pi / 2)$ and $m \in \mathbb{N}$. For each $\theta \in(\omega, \pi / 2)$ and nondegenerate $\psi \in \Psi\left(S_{\theta}^{o}\right)$, let $H_{\mathcal{D}, \psi}^{2}(\mathcal{V})$ denote the set $\overline{\mathrm{R}(\mathcal{D})}$ together with the norm $\|u\|_{H_{\mathcal{D}, \psi}^{2}}:=\|u\|_{E_{\mathcal{D}, \psi}^{2}}$.

When $p \in[1,2)$, we do not know whether or not the completion of $E_{\mathcal{D}, \psi}^{p}(\mathcal{V})$ in $L^{p}(\mathcal{V})$ always exists, so we proceed under additional hypotheses on $\mathcal{D}$. We begin by recording a routine extension of Theorem 4.9 and Lemma 5.2 in [9]. In particular, the improved $\Psi\left(S_{\theta}^{o}\right)$ class exponents in the theorem below follow from (3.9) (for details, see [23], Proposition 7.5, or [12], Theorem 6.2).

Theorem 3.6. Suppose that $M$ is a doubling metric measure space satisfying $\left(\mathrm{D}_{\kappa}\right)$ and that $\mathcal{D}$ satisfies $(\mathrm{H} 1)-(\mathrm{H} 3)$ on $L^{2}(\mathcal{V})$ for some $\omega \in[0, \pi / 2)$ and $m \in \mathbb{N}$. If $p \in[1,2], \theta \in(\omega, \pi / 2), \beta>\kappa / 2 m, \varphi \in \Psi_{\beta}\left(S_{\theta}^{o}\right), \psi \in \Psi_{\beta}\left(S_{\theta}^{o}\right)$ and $\tilde{\psi} \in \Psi^{\beta}\left(S_{\theta}^{o}\right)$, then

$$
\left\|\mathcal{Q}_{\tilde{\psi}}^{\mathcal{D}} \mathcal{S}_{\psi}^{\mathcal{D}} U\right\|_{T^{p}} \lesssim\|U\|_{T^{p}} \quad \forall U \in T^{p} \cap T^{2} .
$$

If, in addition, all of $\varphi, \psi$ and $\tilde{\psi}$ are nondegenerate, then

$$
\mathcal{S}_{\varphi}^{\mathcal{D}}\left(T^{p} \cap T^{2}\right)=\mathcal{S}_{\psi}^{\mathcal{D}}\left(T^{p} \cap T^{2}\right)=\left\{u \in \overline{R(\mathcal{D})}: \mathcal{Q}_{\tilde{\psi}}^{\mathcal{D}} u \in T^{p}\right\}
$$

with the norm equivalence

$$
\|u\|_{E_{\mathcal{D}, \varphi}^{p}} \approx\|u\|_{E_{\mathcal{D}, \psi}^{p}} \approx\left\|\mathcal{Q}_{\tilde{\psi}}^{\mathcal{D}} u\right\|_{T^{p}} \quad \forall u \in E_{\mathcal{D}, \varphi}^{p}=\mathcal{S}_{\varphi}^{\mathcal{D}}\left(T^{p} \cap T^{2}\right) .
$$


If, in addition, the completion $H_{\mathcal{D}, \varphi}^{p}$ of $E_{\mathcal{D}, \varphi}^{p}$ in $L^{p}$ exists, then there are unique extensions $\widetilde{\mathcal{S}}_{\psi}^{\mathcal{D}} \in \mathcal{L}\left(T^{p}, H_{\mathcal{D}, \varphi}^{p}\right)$ and $\widetilde{\mathcal{Q}}_{\tilde{\psi}}^{\mathcal{D}} \in \mathcal{L}\left(H_{\mathcal{D}, \varphi}^{p}, T^{p}\right)$ such that $\widetilde{\mathcal{S}}_{\psi}^{\mathcal{D}}=\mathcal{S}_{\psi}^{\mathcal{D}}$ on $T^{p} \cap T^{2}$ and $\widetilde{\mathcal{Q}}_{\tilde{\psi}}^{\mathcal{D}}=\mathcal{Q}_{\tilde{\psi}}^{\mathcal{D}}$ on $E_{\mathcal{D}, \varphi}^{p}$. It also holds that $H_{\mathcal{D}, \varphi}^{p}=\widetilde{\mathcal{S}}_{\psi}^{\mathcal{D}}\left(T^{p}\right)$ with the norm equivalence

$$
\|u\|_{H_{\mathcal{D}, \varphi}^{p}} \approx \inf \left\{\|U\|_{T^{p}}: U \in T^{p} \text { and } u=\widetilde{\mathcal{S}}_{\psi}^{\mathcal{D}} U\right\} \approx\left\|\widetilde{\mathcal{Q}}_{\tilde{\psi}^{\mathcal{D}}}^{\mathcal{D}}\right\|_{T^{p}} \quad \forall u \in H_{\mathcal{D}, \varphi}^{p} .
$$

Moreover, if $E_{\mathcal{D}, \varphi}^{p}$ is dense in $H_{\mathcal{D}, \varphi}^{p} \cap L^{2}$, then also $\widetilde{\mathcal{Q}}_{\tilde{\psi}}^{\mathcal{D}}=\mathcal{Q}_{\tilde{\psi}}^{\mathcal{D}}$ on $H_{\mathcal{D}, \varphi}^{p} \cap L^{2}$.

Proof. As explained in the remarks preceding the theorem, properties (3.13)-(3.15) are a routine extension of Theorem 4.9 and Lemma 5.2 in [9]. Now suppose that all of $\varphi, \psi$ and $\tilde{\psi}$ are nondegenerate and that the completion $H_{\mathcal{D}, \varphi}^{p}$ of $E_{\mathcal{D}, \varphi}^{p}$ in $L^{p}$ exists. The existence of the completion in $L^{p}$ is used here to ensure that the space $H_{\mathcal{D}, \varphi}^{p} \cap L^{2}$ is a well-defined subspace of, for example, $L_{\text {loc }}^{1}$. It follows from $(3.15)$ that $\left\|\mathcal{S}_{\psi}^{\mathcal{D}} U\right\|_{E_{\mathcal{D}, \varphi}^{p}} \leq\|U\|_{T^{p}}$ for all $U \in T^{p} \cap T^{2}$, and so the operator $\mathcal{S}_{\psi}^{\mathcal{D}}$ in $\mathcal{L}\left(T^{2}, L^{2}\right)$ extends by density to a unique operator $\widetilde{\mathcal{S}}_{\psi}^{\mathcal{D}}$ in $\mathcal{L}\left(T^{p}, H_{\mathcal{D}, \varphi}^{p}\right)$. It follows from $(3.15)$ that the operator $\mathcal{Q}_{\tilde{\psi}}^{\mathcal{D}}$ in $\mathcal{L}\left(L^{2}, T^{2}\right)$ restricts to an operator in $\mathcal{L}\left(E_{\mathcal{D}, \varphi}^{p}, T^{p}\right)$, and so the density of $E_{\mathcal{D}, \varphi}^{p}$ in $H_{\mathcal{D}, \varphi}^{p}$ provides the unique operator $\widetilde{\mathcal{Q}}_{\tilde{\psi}}^{\mathcal{D}}$ in $\mathcal{L}\left(H_{\mathcal{D}, \varphi}^{p}, T^{p}\right)$ such that $\widetilde{\mathcal{Q}}_{\tilde{\psi}}^{\mathcal{D}}=\mathcal{Q}_{\tilde{\psi}}^{\mathcal{D}}$ on $E_{\mathcal{D}, \varphi}^{p}$. We obtain $H_{\mathcal{D}, \varphi}^{p}=\widetilde{\mathcal{S}}_{\psi}^{\mathcal{D}}\left(T^{p}\right)$ and $(3.16)$ by using $\widetilde{\mathcal{S}}_{\psi}^{\mathcal{D}}$ and $\widetilde{\mathcal{Q}}_{\tilde{\psi}}^{\mathcal{D}}$ to extend Proposition 3.3 and properties $(3.13)-(3.15)$. Finally, if $E_{\mathcal{D}, \varphi}^{p}$ is dense in $H_{\mathcal{D}, \varphi}^{p} \cap L^{2}$, then for each $u$ in $H_{\mathcal{D}, \varphi}^{p} \cap L^{2}$, there exists a sequence $\left(u_{n}\right)_{n}$ in $E_{\mathcal{D}, \varphi}^{p}$ such that $u_{n}$ converges to $u$ in both $H_{\mathcal{D}, \varphi}^{p}$ and $L^{2}$, so by writing

$\left\|\widetilde{\mathcal{Q}}_{\tilde{\psi}}^{\mathcal{D}} u-\mathcal{Q}_{\tilde{\psi}}^{\mathcal{D}} u\right\|_{T^{p}+T^{2}} \leq\left\|\widetilde{\mathcal{Q}}_{\tilde{\psi}}^{\mathcal{D}} u-\mathcal{Q}_{\tilde{\psi}}^{\mathcal{D}} u_{n}\right\|_{T^{p}}+\left\|\mathcal{Q}_{\tilde{\psi}}^{\mathcal{D}} u_{n}-\mathcal{Q}_{\tilde{\psi}}^{\mathcal{D}} u\right\|_{T^{2}} \lesssim\left\|u-u_{n}\right\|_{H_{\mathcal{D}, \varphi}^{p} \cap L^{2}}$

we conclude that $\widetilde{\mathcal{Q}}_{\tilde{\psi}}^{\mathcal{D}} u=\mathcal{Q}_{\tilde{\psi}}^{\mathcal{D}} u$. This completes the proof.

Remark 3.7. In the context of Theorem 3.6, if the completion $H_{\mathcal{D}, \varphi}^{p}(\mathcal{V})$ of $E_{\mathcal{D}, \varphi}^{p}(\mathcal{V})$ in $L^{p}(\mathcal{V})$ exists for some nondegenerate $\varphi \in \Psi_{\beta}\left(S_{\theta}^{o}\right)$, then (3.16) implies that the completion $H_{\mathcal{D}, \psi}^{p}(\mathcal{V})$ of $E_{\mathcal{D}, \psi}^{p}(\mathcal{V})$ in $L^{p}(\mathcal{V})$ exists for all nondegenerate $\psi \in \Psi_{\beta}\left(S_{\theta}^{o}\right)$. Therefore, we could adopt the notation in [9] whereby $H_{\mathcal{D}}^{p}(\mathcal{V})$ denotes any of the equivalent Banach spaces $H_{\mathcal{D}, \psi}^{p}(\mathcal{V})$. We found it convenient not to do this, however, given the technical nature of this article.

We now introduce atoms and molecules in order to show that $E_{\mathcal{D}, \psi}^{p}(\mathcal{V}) \subseteq L^{p}(\mathcal{V})$.

Definition 3.8. Suppose that $\mathcal{D}$ satisfies $(\mathrm{H} 1)$ and $(\mathrm{H} 3)$ on $L^{2}(\mathcal{V})$ for some $m \in \mathbb{N}$. For $N \in \mathbb{N}$, a section $a \in L^{2}(\mathcal{V})$ is called an $H_{\mathcal{D}}^{1}(\mathcal{V})$-molecule of type $N$ when there exist a section $b \in \operatorname{Dom}\left(\mathcal{D}^{N}\right)$ and a ball $B \subseteq M$ of radius $r(B)>0$ such that $a=\mathcal{D}^{N} b$ and the following hold for all $k \in \mathbb{N}_{0}$ :

(1) $\left\|\mathbf{1}_{k}(B) a\right\|_{2} \leq 2^{-k} \mu\left(2^{k} B\right)^{-1 / 2}$;

(2) $\left\|\mathbf{1}_{k}(B) b\right\|_{2} \leq r(B)^{m N} 2^{-k} \mu\left(2^{k} B\right)^{-1 / 2}$, 
where $\mathbf{1}_{0}(B)=\mathbf{1}_{B}$ and $\mathbf{1}_{k}(B)=\mathbf{1}_{2^{k} B \backslash 2^{k-1} B}$ for $k \in \mathbb{N}$. An $H_{\mathcal{D}}^{1}(\mathcal{V})$-atom of type $N$ is defined in the same way, except that $a$ and $b$ are required to be supported on the ball $B$, which obviates (1) and (2) when $k \geq 1$.

The proof below uses a molecular characterisation obtained in [9], Section 6.1.

Lemma 3.9. Suppose that $M$ is a doubling metric measure space satisfying $\left(\mathrm{D}_{\kappa}\right)$ and that $\mathcal{D}$ satisfies $(\mathrm{H} 1)-(\mathrm{H} 3)$ on $L^{2}(\mathcal{V})$ for some $\omega \in[0, \pi / 2)$ and $m \in \mathbb{N}$. If $p \in[1,2], \theta \in(\omega, \pi / 2), \beta>\kappa / 2 m$ and $\psi \in \Psi_{\beta}\left(S_{\theta}^{o}\right)$ is nondegenerate, then $E_{\mathcal{D}, \psi}^{p}(\mathcal{V}) \subseteq L^{p}(\mathcal{V})$

Proof. When $p=2$, the result holds by (3.12). When $p \in[1,2)$, it suffices to prove the result for a fixed nondegenerate $\psi$ in $\Psi_{\beta}\left(S_{\theta}^{o}\right)$ by $(3.15)$. Therefore, we fix $N \in \mathbb{N}$ and use the construction in [9], Lemma 6.7, to fix a nondegenerate $\psi$ in $\Psi_{\beta}\left(S_{\theta}^{o}\right)$ such that $\mathcal{S}_{\psi}^{\mathcal{D}}(A)$ is an $H_{\mathcal{D}}^{1}$-molecule of type $N$ whenever $A$ is a $T^{1}$-atom.

Now consider when $p=1$. For all $H_{\mathcal{D}}^{1}$-molecules $a$ of type $N$, note that

$$
\|a\|_{1} \leq \sum_{k=0}^{\infty} \mu\left(2^{k} B\right)^{1 / 2}\left\|\mathbf{1}_{k}(B) a\right\|_{2} \leq 2 .
$$

Suppose that $u \in E_{\mathcal{D}, \psi}^{1}$ and $V \in T^{1} \cap T^{2}$ such that $u=\mathcal{S}_{\psi}^{\mathcal{D}} V$ and $\|V\|_{T^{1}} \leq$ $2\|u\|_{E_{\mathcal{D}, \psi}^{1}}$. The atomic characterisation of $T^{1}$ in Theorem 3.1 provides a sequence $\left(\lambda_{j}\right)_{j}$ in $\ell^{1}$ and a sequence $\left(A_{j}\right)_{j}$ of $T^{1}$-atoms such that $\sum_{j} \lambda_{j} A_{j}$ converges to $V$ in $T^{1}$ and $T^{2}$, and $\left\|\left(\lambda_{j}\right)_{j}\right\|_{\ell^{1}} \approx\|V\|_{T^{1}}$. The operator $\mathcal{S}_{\psi}^{\mathcal{D}}$ in $\mathcal{L}\left(T^{2}, L^{2}\right)$ is bounded from $\left(T^{1} \cap T^{2},\|\cdot\|_{T^{1}}\right)$ into $E_{\mathcal{D}, \psi}^{1}$, by the definition of $E_{\mathcal{D}, \psi}^{1}$, so $\sum_{j} \lambda_{j} \mathcal{S}_{\psi}^{\mathcal{D}} A_{j}$ converges to $u$ in $E_{\mathcal{D}, \psi}^{1}$ and $L^{2}$. Now recall that $\psi$ has the property whereby each $\mathcal{S}_{\psi}^{\mathcal{D}} A_{j}$ is an $H_{\mathcal{D}}^{1}$-molecule of type $N$, so in accordance with (3.17), the sequence $\left(\mathcal{S}_{\psi}^{\mathcal{D}} A_{j}\right)_{j}$ is uniformly bounded in $L^{1}$, and as such, there exists $\tilde{u}$ in $L^{1}$ such that $\sum_{j} \lambda_{j} \mathcal{S}_{\psi}^{\mathcal{D}} A_{j}$ converges to $\tilde{u}$ in $L^{1}$. We must have $u=\tilde{u} \in L^{1}$, since $L^{1}$ and $L^{2}$ are embedded in $L_{\text {loc }}^{1}$, and so $\sum_{j} \lambda_{j} \mathcal{S}_{\psi}^{\mathcal{D}} A_{j}$ converges to $u$ in $L^{1}$ with $\|u\|_{1}=\lim _{n \rightarrow \infty}\left\|\sum_{j=1}^{n} \lambda_{j} \mathcal{S}_{\psi}^{\mathcal{D}} A_{j}\right\|_{1} \lesssim\left\|\left(\lambda_{j}\right)_{j}\right\|_{\ell^{1}} \lesssim\|V\|_{T^{1}} \lesssim\|u\|_{E_{\mathcal{D}, \psi}^{1}}$. This completes the proof when $p=1$.

Now consider when $p \in(1,2)$. We have shown that $E_{\mathcal{D}, \psi}^{1} \subseteq L^{1}$, so by the definition of $E_{\mathcal{D}, \psi}^{1}$, it follows that $\left\|\mathcal{S}_{\psi}^{\mathcal{D}} U\right\|_{1} \lesssim\left\|\mathcal{S}_{\psi}^{\mathcal{D}} U\right\|_{E_{\mathcal{D}, \psi}^{1}} \leq\|U\|_{T^{1}}$ for all $U \in$ $T^{1} \cap T^{2}$. Therefore, the operator $\mathcal{S}_{\psi}^{\mathcal{D}}$ in $\mathcal{L}\left(T^{2}, L^{2}\right)$ has an extension in $\mathcal{L}\left(T^{1}, L^{1}\right)$, and then by the interpolation of tent spaces in (3.11), this extension is also in $\mathcal{L}\left(T^{p}, L^{p}\right)$. It follows that $E_{\mathcal{D}, \psi}^{p} \subseteq L^{p}$, since for each $u \in E_{\mathcal{D}, \psi}^{p}$, there exists $V \in T^{p} \cap T^{2}$ such that $u=\mathcal{S}_{\psi}^{\mathcal{D}} V$ and $\|V\|_{T^{p}} \leq 2\|u\|_{E_{\mathcal{D}, \psi}^{p}}$, hence $\|u\|_{p}=\left\|\mathcal{S}_{\psi}^{\mathcal{D}} V\right\|_{p} \lesssim$ $\|V\|_{T^{p}} \lesssim\|u\|_{E_{\mathcal{D}, \psi}^{p}}$.

The proof of Lemma 3.9 shows that for each $N \in \mathbb{N}$ and $u \in E_{\mathcal{D}, \psi}^{1}(\mathcal{V})$, there exist a sequence $\left(\lambda_{j}\right)_{j}$ in $\ell^{1}$ and a sequence $\left(a_{j}\right)_{j}$ of $H_{\mathcal{D}}^{1}(\mathcal{V})$-molecules of type $N$ such that $\sum_{j} \lambda_{j} a_{j}$ converges to $u$ in $E_{\mathcal{D}, \psi}^{1}(\mathcal{V})$ and $L^{1}(\mathcal{V})$ with $\left\|\left(\lambda_{j}\right)_{j}\right\|_{\ell^{1}} \lesssim\|u\|_{E_{\mathcal{D}, \psi}^{1}}$. Although 
this characterisation extends to completions of $E_{\mathcal{D}, \psi}^{1}(\mathcal{V})$ (see Theorem 3.13), it does not seem to guarantee that the completion of $E_{\mathcal{D}, \psi}^{1}(\mathcal{V})$ in $L^{1}(\mathcal{V})$ exists. We introduce hypothesis $(\mathrm{H} 4)_{\Psi}$ on $\mathcal{D}$ in the next theorem for this reason.

Theorem 3.10. Suppose that $M$ is a doubling metric measure space satisfying $\left(\mathrm{D}_{\kappa}\right)$ and that $\mathcal{D}$ satisfies $(\mathrm{H} 1)-(\mathrm{H} 3)$ on $L^{2}(\mathcal{V})$ for some $\omega \in[0, \pi / 2)$ and $m \in \mathbb{N}$. If $1 \leq q \leq p \leq 2, \theta \in(\omega, \pi / 2), \beta>\kappa / 2 m, \psi \in \Psi_{\beta}\left(S_{\theta}^{o}\right)$ is nondegenerate and

there exists a nondegenerate function $\tilde{\psi} \in \Psi^{\beta}\left(S_{\theta}^{o}\right)$ such that the set
$(\mathrm{H} 4)_{\Psi} \quad\left\{F \in T^{2} \cap T^{q^{\prime}}: \mathcal{S}_{\tilde{\psi}^{*}}^{\mathcal{D}^{*}} F \in L^{q^{\prime}}(\mathcal{V})\right\}$ is weak-star dense in $T^{p^{\prime}}\left(\mathcal{V}_{+}\right)$

where $1 / q+1 / q^{\prime}=1$, then the completion $H_{\mathcal{D}, \psi}^{p}(\mathcal{V})$ of $E_{\mathcal{D}, \psi}^{p}(\mathcal{V})$ in $L^{p}(\mathcal{V})$ exists. Moreover, it holds that $H_{\mathcal{D}, \psi}^{p}(\mathcal{V}) \cap L^{2}(\mathcal{V})=E_{\mathcal{D}, \psi}^{p}(\mathcal{V})$.

Proof. Lemma 3.9 shows that $E_{\mathcal{D}, \psi}^{p} \subseteq L^{p}$, so the existence of the completion of $E_{\mathcal{D}, \psi}^{p}$ in $L^{p}$ will follow by proving (3) in Proposition (2.2) with $X=E_{\mathcal{D}, \psi}^{p}$ and $Y=L^{p}$. To this end, let $\left(u_{n}\right)_{n}$ denote a Cauchy sequence in $E_{\mathcal{D}, \psi}^{p}$ that converges to 0 in $L^{p}$. We claim that $\left(u_{n}\right)_{n}$ converges to 0 in $E_{\mathcal{D}, \psi}^{p}$. To see this, fix $\tilde{\psi}$ in $\Psi^{\beta}\left(S_{\theta}^{o}\right)$ satisfying $(\mathrm{H} 4)_{\Psi}$ so that $\mathcal{E}:=\left\{F \in T^{2} \cap T^{q^{\prime}}: \mathcal{S}_{\tilde{\psi}^{*}}^{\mathcal{D}^{*}} F \in L^{q^{\prime}}\right\}$ is weak-star dense in $T^{p^{\prime}}$. For all $n \in \mathbb{N}$, we have by (3.15) that

$$
\left\|u_{n}\right\|_{E_{\mathcal{D}, \psi}^{p}} \approx\left\|\mathcal{Q}_{\tilde{\psi}}^{\mathcal{D}} u_{n}\right\|_{T^{p}}
$$

and since $\left(u_{n}\right)_{n}$ is Cauchy in $E_{\mathcal{D}, \psi}^{p}$, there exists $U$ in $T^{p}$ such that $\mathcal{Q}_{\tilde{\psi}}^{\mathcal{D}} u_{n}$ converges to $U$ in $T^{p}$. Using the duality pairing in (3.10), for all $n \in \mathbb{N}$ and $F \in \mathcal{E}$, we have

$$
\begin{aligned}
\left|\langle U, F\rangle_{T^{2}}\right| & \leq\left|\left\langle U-\mathcal{Q}_{\tilde{\psi}}^{\mathcal{D}} u_{n}, F\right\rangle_{T^{2}}\right|+\left|\left\langle\mathcal{Q}_{\tilde{\psi}}^{\mathcal{D}} u_{n}, F\right\rangle_{T^{2}}\right| \\
& \lesssim\left\|U-\mathcal{Q}_{\tilde{\psi}}^{\mathcal{D}} u_{n}\right\|_{T^{p}}\|F\|_{T^{p^{\prime}}}+\left\|u_{n}\right\|_{L^{p}}\left\|\mathcal{S}_{\tilde{\psi}^{*}}^{\mathcal{D}^{*}} F\right\|_{L^{p^{\prime}}}
\end{aligned}
$$

since $2 \leq p^{\prime} \leq q^{\prime}$ ensures that $L^{2} \cap L^{q^{\prime}} \subseteq L^{p^{\prime}}$ and $T^{2} \cap T^{q^{\prime}} \subseteq T^{p^{\prime}}$. Moreover, since $\left\|\mathcal{S}_{\tilde{\psi}^{*}}^{\mathcal{D}^{*}} F\right\|_{L^{p^{\prime}}}<\infty$ and $\|F\|_{T^{p^{\prime}}}<\infty$, the preceding convergence results imply that

$$
\langle U, F\rangle_{T^{2}}=0 \quad \forall F \in \mathcal{E} .
$$

Then, since $U \in T^{p}$ and $\mathcal{E}$ is weak-star dense in $T^{p^{\prime}}$, it follows that $\langle U, F\rangle_{T^{2}}=0$ for all $F \in T^{p^{\prime}}$, hence $U=0$ and $\left(u_{n}\right)_{n}$ converges to 0 in $E_{\mathcal{D}, \psi}^{p}$, as claimed. This proves that the completion $H_{\mathcal{D}, \psi}^{p}$ of $E_{\mathcal{D}, \psi}^{p}$ in $L^{p}$ exists.

The inclusion $E_{\mathcal{D}, \psi}^{p} \subseteq H_{\mathcal{D}, \psi}^{p} \cap L^{2}$ holds by (3.14). To prove the reverse inclusion, suppose that $u \in H_{\mathcal{D}, \psi}^{p} \cap L^{2}$. The density of $E_{\mathcal{D}, \psi}^{p}$ in $H_{\mathcal{D}, \psi}^{p}$ provides a sequence $\left(u_{n}\right)_{n}$ in $E_{\mathcal{D}, \psi}^{p}$ that converges to $u$ in $H_{\mathcal{D}, \psi}^{p}$. This sequence also converges in $L^{p}$ because $H_{\mathcal{D}, \psi}^{p} \subseteq L^{p}$. Moreover, since (3.18) holds and $\left(u_{n}\right)_{n}$ is Cauchy in $E_{\mathcal{D}, \psi}^{p}$, there exists $U$ in $T^{p}$ such that $\mathcal{Q}_{\tilde{\psi}}^{\mathcal{D}} u_{n}$ converges to $U$ in $T^{p}$. For all $n \in \mathbb{N}$ and $F \in \mathcal{E}$, 
we have

$$
\begin{aligned}
\left|\left\langle U-\mathcal{Q}_{\tilde{\psi}}^{\mathcal{D}} u, F\right\rangle_{T^{2}}\right| & \leq\left|\left\langle U-\mathcal{Q}_{\tilde{\psi}}^{\mathcal{D}} u_{n}, F\right\rangle_{T^{2}}\right|+\left|\left\langle\mathcal{Q}_{\tilde{\psi}}^{\mathcal{D}} u_{n}-\mathcal{Q}_{\tilde{\psi}}^{\mathcal{D}} u, F\right\rangle_{T^{2}}\right| \\
& \lesssim\left\|U-\mathcal{Q}_{\tilde{\psi}}^{\mathcal{D}} u_{n}\right\|_{T^{p}}\|F\|_{T^{p^{\prime}}}+\left\|u_{n}-u\right\|_{p}\left\|\mathcal{S}_{\tilde{\psi}^{*}}^{\mathcal{D}^{*}} F\right\|_{p^{\prime}}
\end{aligned}
$$

The preceding convergence arguments then show that $U=\mathcal{Q}_{\tilde{\psi}}^{\mathcal{D}} u \in T^{p} \cap T^{2}$, and since $\left\|\mathcal{Q}_{\tilde{\psi}}^{\mathcal{D}} u\right\|_{T^{p}} \approx\|u\|_{E_{\mathcal{D}, \psi}^{p}}$, we conclude that $u \in E_{\mathcal{D}, \psi}^{p}$, as required.

Remark 3.11. In the context of Theorem 3.10, since $M$ is $\sigma$-finite, hypothesis $(\mathrm{H} 4)_{\Psi}$ holds whenever $\mathcal{S}_{\tilde{\psi}^{*}}^{\mathcal{D}^{*}}\left(T_{0}^{2}\left(\mathcal{V}_{+}\right)\right) \subseteq L^{q^{\prime}}(\mathcal{V})$, where $T_{0}^{2}\left(\mathcal{V}_{+}\right)$denotes the set of all $U$ in $T^{2}\left(\mathcal{V}_{+}\right)$for which there exists some ball $B$ in $M$ and some constants $b>a>0$ such that $\operatorname{sppt}(U) \subseteq B \times[a, b]$. This is because $T_{0}^{2}\left(\mathcal{V}_{+}\right)$is weak-star dense in $T^{p^{\prime}}\left(\mathcal{V}_{+}\right)$for all $p \in[1,2]$. To see this, let $\left(B_{n}\right)_{n}$ denote an increasing sequence of balls that exhaust $M$. For all $F \in T^{p}\left(\mathcal{V}_{+}\right)$and all $G \in T^{p^{\prime}}\left(\mathcal{V}_{+}\right)$, we have

$$
\int_{0}^{\infty} \int_{M}\left|\left\langle F_{t}(x), G_{t}(x)\right\rangle_{x}\right| d \mu(x) \frac{d t}{t} \lesssim\|F\|_{T^{p}}\|G\|_{T^{p^{\prime}}}
$$

by the duality in (3.10). The dominated convergence theorem then implies that $\left\langle F, \mathbf{1}_{B_{n} \times[1 / n, n]} G\right\rangle_{T^{2}}$ converges to $\langle F, G\rangle_{T^{2}}$, which proves the asserted weak-star density, since $\mathbf{1}_{B_{n} \times[1 / n, n]} G \in T_{0}^{2}\left(\mathcal{V}_{+}\right)$.

\subsection{Molecular theory}

We defined $H_{\mathcal{D}}^{1}(\mathcal{V})$-molecules and atoms in Definition 3.8. The molecular characterisation of $H_{\mathcal{D}, \psi}^{1}(\mathcal{V})$ below is based on the characterisation obtained in Theorem 6.2 of [9]. It is convenient to first introduce the following spaces.

Definition 3.12. Suppose that $\mathcal{D}$ satisfies $(\mathrm{H} 1)$ and $(\mathrm{H} 3)$ on $L^{2}(\mathcal{V})$ for some $m \in \mathbb{N}$. For $N \in \mathbb{N}$, the Banach space $H_{\mathcal{D}, \operatorname{mol}(N)}^{1}(\mathcal{V})$ is the set of all $u$ in $L^{1}(\mathcal{V})$ for which there exist a sequence $\left(\lambda_{j}\right)_{j}$ in $\ell^{1}$ and a sequence $\left(a_{j}\right)_{j}$ of $H_{\mathcal{D}}^{1}$-molecules of type $N$ such that $\sum_{j} \lambda_{j} a_{j}$ converges to $u$ in $L^{1}(\mathcal{V})$, together with the norm

$$
\|u\|_{H_{\mathcal{D}, \operatorname{mol}(N)}^{1}}:=\inf \left\{\left\|\left(\lambda_{j}\right)_{j}\right\|_{\ell^{1}}: \sum_{j} \lambda_{j} a_{j} \text { converges to } u \text { in } L^{1}\right\} .
$$

The Banach space $H_{\mathcal{D}, \text { at }(N)}^{1}(\mathcal{V})$ is defined by replacing molecules with atoms.

The $L^{1}(\mathcal{V})$ convergence in the above definition ensures that $H_{\mathcal{D}, \operatorname{mol}(N)}^{1}(\mathcal{V})$ and $H_{\mathcal{D}, \text { at }(N)}^{1}(\mathcal{V})$ are complete. This is because molecules and atoms are uniformly bounded in $L^{1}(\mathcal{V})$. In particular, if $\left(u_{n}\right)_{n}$ is a sequence in $H_{\mathcal{D}, \operatorname{mol}(N)}^{1}(\mathcal{V})$ such that $\sum_{n}\left\|u_{n}\right\|_{H_{\mathcal{D}, \operatorname{mol}(N)}^{1}}$ is finite, then the uniform $L^{1}(\mathcal{V})$ bound for molecules and the dominated convergence theorem imply that $\sum_{n} u_{n}$ converges in the $H_{\mathcal{D}, \operatorname{mol}(N)}^{1}(\mathcal{V})$ norm to some $u \in H_{\mathcal{D}, \operatorname{mol}(N)}^{1}(\mathcal{V})$, hence $H_{\mathcal{D}, \operatorname{mol}(N)}^{1}(\mathcal{V})$ is complete. The $L^{1}(\mathcal{V})$ convergence requirement also distinguishes these spaces from those in the literature that are defined as an abstract completion of a molecular or atomic space on which $L^{2}(\mathcal{V})$ convergence is required. This is discussed further in Remark 3.15. 
The embedding $H_{\mathcal{D}, \psi}^{1}(\mathcal{V}) \subseteq L^{1}(\mathcal{V})$ is not required to define the molecular space nor the atomic space, since $H_{\mathcal{D} \text {, at }(N)}^{1}(\mathcal{V}) \subseteq H_{\mathcal{D}, \operatorname{mol}(N)}^{1}(\mathcal{V}) \subseteq L^{1}(\mathcal{V})$ is automatic. It is only when the embedding of $H_{\mathcal{D}, \psi}^{1}(\mathcal{V})$ in $L^{1}(\mathcal{V})$ holds, however, that we can establish the following connection.

Theorem 3.13. Suppose that $M$ is a doubling metric measure space satisfying $\left(\mathrm{D}_{\kappa}\right)$ and that $\mathcal{D}$ satisfies $(\mathrm{H} 1)-(\mathrm{H} 3)$ on $L^{2}(\mathcal{V})$ for some $\omega \in[0, \pi / 2)$ and $m \in \mathbb{N}$. Also, assume that for some $\theta \in(\omega, \pi / 2), \beta>\kappa / 2 m$ and nondegenerate $\psi \in \Psi_{\beta}\left(S_{\theta}^{o}\right)$, the completion $H_{\mathcal{D}, \psi}^{1}(\mathcal{V})$ of $E_{\mathcal{D}, \psi}^{1}(\mathcal{V})$ in $L^{1}(\mathcal{V})$ exists. It follows that if $N \in \mathbb{N}$ and $N>\kappa / 2 m$, then $H_{\mathcal{D}, \psi}^{1}(\mathcal{V})=H_{\mathcal{D}, \operatorname{mol}(N)}^{1}(\mathcal{V})$.

Proof. Suppose that $N \in \mathbb{N}$. The proof that $H_{\mathcal{D}, \psi}^{1} \subseteq H_{\mathcal{D}, \operatorname{mol}(N)}^{1}$ follows that of Lemma 3.9, except we need to replace $L^{2}$ convergence with $H_{\mathcal{D}, \psi}^{1}$ convergence. We use the construction in [9], Lemma 6.7 , to fix a nondegenerate $\tilde{\psi}$ in $\Psi_{\beta}\left(S_{\theta}^{o}\right)$ such that $\mathcal{S}_{\tilde{\psi}}^{\mathcal{D}} A$ is an $H_{\mathcal{D}}^{1}$-molecule of type $N$ whenever $A$ is a $T^{1}$-atom. The existence of the completion $H_{\mathcal{D}, \psi}^{1}$ of $E_{\mathcal{D}, \psi}^{1}$ in $L^{1}$ allows us to apply (3.16) and ensures that $H_{\mathcal{D}, \psi}^{1} \subseteq L^{1}$. Suppose that $u \in H_{\mathcal{D}, \psi}^{1}$ and use (3.16) to choose $V$ in $T^{1}$ such that $u=\widetilde{\mathcal{S}}_{\tilde{\psi}}^{\mathcal{D}} V$ and $\|V\|_{T^{1}} \leq 2\|u\|_{H_{\mathcal{D}, \psi}^{1}}$. The atomic characterisation of $T^{1}$ in Theorem 3.1 provides a sequence $\left(\lambda_{j}\right)_{j}$ in $\ell^{1}$ and a sequence $\left(A_{j}\right)_{j}$ of $T^{1}$-atoms such that $\sum_{j} \lambda_{j} A_{j}$ converges to $V$ in $T^{1}$ and $\left\|\left(\lambda_{j}\right)_{j}\right\|_{\ell^{1}} \lesssim\|V\|_{T^{1}}$. It follows that $\sum_{j} \lambda_{j} \mathcal{S}_{\tilde{\psi}}^{\mathcal{D}} A_{j}$ converges to $u$ in $H_{\mathcal{D}, \psi}^{1}$, and in $L^{1}$, because $\widetilde{\mathcal{S}}_{\tilde{\psi}}^{\mathcal{D}} \in \mathcal{L}\left(T^{1}, H_{\mathcal{D}, \psi}^{1}\right)$ and $\widetilde{\mathcal{S}}_{\tilde{\psi}}^{\mathcal{D}}=\mathcal{S}_{\tilde{\psi}}^{\mathcal{D}}$ on $T^{1} \cap T^{2}$ by $(3.16)$, and because $H_{\mathcal{D}, \psi}^{1} \subseteq L^{1}$. Now recall that $\tilde{\psi}$ has the property whereby each $\mathcal{S}_{\tilde{\psi}}^{\mathcal{D}} A_{j}$ is an $H_{\mathcal{D}}^{1}$-molecule of type $N$, so then $u \in H_{\mathcal{D}, \operatorname{mol}(N)}^{1}$ and $\|u\|_{H_{\mathcal{D}, \operatorname{mol}(N)}^{1}} \leq\left\|\left(\lambda_{j}\right)_{j}\right\|_{\ell^{1}} \lesssim\|V\|_{T^{1}} \lesssim\|u\|_{H_{\mathcal{D}, \psi}^{1}}$, hence $H_{\mathcal{D}, \psi}^{1} \subseteq H_{\mathcal{D}, \operatorname{mol}(N)}^{1}$.

Now suppose that $N \in \mathbb{N}, N>\kappa / 2 m$ and $u \in H_{\mathcal{D}, \operatorname{mol}(N)}^{1}$. Then $u \in L^{1}$ and there is a sequence $\left(\lambda_{j}\right)_{j}$ in $\ell^{1}$ and a sequence $\left(a_{j}\right)_{j}$ of $H_{\mathcal{D}}^{1}$-molecules of type $N$ such that $\sum_{j} \lambda_{j} a_{j}$ converges to $u$ in $L^{1}$ with $\left\|\left(\lambda_{j}\right)_{j}\right\|_{\ell^{1}} \leq 2\|u\|_{H_{\mathcal{D}, \operatorname{mol}(N)}^{1}}$. The construction in [9], Lemma 6.8, allows us to fix $\tilde{\tilde{\psi}}$ in $\Psi^{\beta}\left(S_{\theta}^{o}\right)$ such that $\mathcal{Q}_{\tilde{\tilde{\psi}}}^{\mathcal{D}}$ is uniformly bounded in $T^{1}$ on all $H_{\mathcal{D}}^{1}$-molecules of type $N$ (this requires $N>\kappa / 2 m$ ), so by (3.15) we have

$$
\left\|\sum_{j=1}^{l} \lambda_{j} a_{j}-\sum_{j=1}^{k} \lambda_{j} a_{j}\right\|_{H_{\mathcal{D}, \psi}^{1}} \lesssim \sum_{j=k+1}^{l}\left|\lambda_{j}\right|\left\|\mathcal{Q}_{\tilde{\psi}}^{\mathcal{D}} a_{j}\right\|_{T^{1}} \lesssim \sum_{j=k+1}^{l}\left|\lambda_{j}\right|
$$

whenever $l>k>0$. Therefore, there exists $v$ in $H_{\mathcal{D}, \psi}^{1}$ such that $\sum_{j} \lambda_{j} a_{j}$ converges to $v$ in $H_{\mathcal{D}, \psi}^{1}$, and hence in $L^{1}$ because $H_{\mathcal{D}, \psi}^{1} \subseteq L^{1}$. It follows that $u=v \in H_{\mathcal{D}, \psi}^{1}$ with

$$
\|u\|_{H_{\mathcal{D}, \psi}^{1}} \lesssim \lim _{k \rightarrow \infty} \sum_{j=1}^{k}\left|\lambda_{j}\right|\left\|\mathcal{Q}_{\tilde{\tilde{\psi}}}^{\mathcal{D}} a_{j}\right\|_{T^{1}} \lesssim\left\|\left(\lambda_{j}\right)_{j}\right\|_{\ell^{1}} \lesssim\|u\|_{H_{\mathcal{D}, \operatorname{mol}(N)}^{1}},
$$

so $H_{\mathcal{D}, \operatorname{mol}(N)}^{1} \subseteq H_{\mathcal{D}, \psi}^{1}$ and the proof is complete. 
Remark 3.14. The proof of Theorem 3.13 shows that the same result holds when the $L^{1}(\mathcal{V})$ convergence required in Definition 3.12 is replaced with $H_{\mathcal{D}, \psi}^{1}(\mathcal{V})$ convergence.

Remark 3.15. If we define $E_{\mathcal{D}, \operatorname{mol}(N)}^{1}(\mathcal{V})$ to be the normed space obtained by replacing $L^{1}(\mathcal{V})$ convergence with $L^{2}(\mathcal{V})$ convergence in Definition 3.12, then we can prove that $E_{\mathcal{D}, \psi}^{1}(\mathcal{V})=E_{\mathcal{D}, \operatorname{mol}(N)}^{1}(\mathcal{V})$ without assuming that the embedding $H_{\mathcal{D}, \psi}^{1}(\mathcal{V}) \subseteq L^{1}(\mathcal{V})$ holds. This was known previously (see Theorem 3.5 in [21]). In particular, the proof of Lemma 3.9 shows that $E_{\mathcal{D}, \psi}^{1}(\mathcal{V}) \subseteq E_{\mathcal{D}, \operatorname{mol}(N)}^{1}(\mathcal{V})$, whilst the reverse inclusion is proved in a manner similar to that of Theorem 3.13. This means that we can identify any completion of $E_{\mathcal{D}, \psi}^{1}(\mathcal{V})$ with any completion of $E_{\mathcal{D}, \operatorname{mol}(N)}^{1}(\mathcal{V})$, but both are still abstract spaces and it is not known whether either can be embedded in $L^{1}(\mathcal{V})$, or in any function space, without the extra hypotheses on $\mathcal{D}$ in Theorem 3.10 (or Theorem 4.7).

\subsection{The embedding $H_{L}^{p} \subseteq L^{p}$ for divergence form elliptic operators}

It is a simple matter to verify the hypotheses of Theorem 3.10 for an operator that generates a semigroup satisfying pointwise kernel estimates. We demonstrate this by obtaining Theorem 1.2 as a special case of the more general result below.

Let $M=\mathbb{R}^{n}$ and consider the divergence form operator $L=-\operatorname{div} A \nabla$ acting on $L^{2}\left(\mathbb{R}^{n}\right)$ and interpreted in the usual weak sense via a sesquilinear form, where $A \in L^{\infty}\left(\mathbb{R}^{n}, \mathcal{L}\left(\mathbb{C}^{n}\right)\right)$ is elliptic in the sense that there exists $\lambda>0$ such that

$$
\operatorname{Re}\langle A(x) \zeta, \zeta\rangle_{\mathbb{C}^{n}} \geq \lambda|\zeta|^{2} \quad \forall \zeta \in \mathbb{C}^{n} \text {, a.e. } x \in \mathbb{R}^{n}
$$

There exists $\omega_{L} \in[0, \pi / 2)$, depending on $\lambda$ and $\|A\|_{\infty}$, such that $L$ is $\omega_{L}$-sectorial (see, for instance, Chapter 2 in [5]), hence $L: \operatorname{Dom}(L) \subseteq L^{2}\left(\mathbb{R}^{n}\right) \rightarrow L^{2}\left(\mathbb{R}^{n}\right)$ satisfies (H1)-(H2) with $\omega=\omega_{L}$. Note that $\operatorname{Dom}(L)=\left\{u \in W^{1,2}\left(\mathbb{R}^{n}\right): A \nabla u \in \operatorname{Dom}\left(\nabla^{*}\right)\right\}$. It is also known that $L$ satisfies (H3) with $m=2$ (see Lemma 2.1 in [6]).

In order to embed $H_{L, \psi}^{p}\left(\mathbb{R}^{n}\right)$ in $L^{p}\left(\mathbb{R}^{n}\right)$ when $1 \leq q \leq p \leq 2$ and $1 / q^{\prime}+1 / q=1$, we assume that there exists $g \in L_{\text {loc }}^{2}((0, \infty))$ such that the analytic semigroup $\left(e^{-t L^{*}}\right)_{t>0}$ generated by the adjoint $-L^{*}$ on $L^{2}\left(\mathbb{R}^{n}\right)$ satisfies

$$
\left\|e^{-t L^{*}} u\right\|_{q^{\prime}} \leq g(t)\|u\|_{2} \quad \forall u \in L^{2}\left(\mathbb{R}^{n}\right), \forall t>0 .
$$

This assumption is always satisfied when $2 n /(n+2) \leq q \leq 2$ in dimension $n \geq 3$ (see Proposition 3.2 in [5] and Lemma 2.25 in [21]). It remains an open question, however, as to whether the following theorem holds in the absence of estimates such as $(3.20)$.

Theorem 3.16. Suppose that $A \in L^{\infty}\left(\mathbb{R}^{n}, \mathcal{L}\left(\mathbb{C}^{n}\right)\right)$ is elliptic and $L=-\operatorname{div} A \nabla$ on $L^{2}\left(\mathbb{R}^{n}\right)$ satisfies (3.20) for some $q \in[1,2]$. If $q \leq p \leq 2, \theta \in\left(\omega_{L}, \pi / 2\right), \beta>n / 4$ and $\psi \in \Psi_{\beta}\left(S_{\theta}^{o}\right)$ is nondegenerate, then the completion $H_{L, \psi}^{p}\left(\mathbb{R}^{n}\right)$ of $E_{L, \psi}^{p}\left(\mathbb{R}^{n}\right)$ in $L^{p}\left(\mathbb{R}^{n}\right)$ exists and $H_{L, \psi}^{p}\left(\mathbb{R}^{n}\right) \cap L^{2}\left(\mathbb{R}^{n}\right)=E_{L, \psi}^{p}\left(\mathbb{R}^{n}\right)$. Moreover, if $q=1, N \in \mathbb{N}$ and $N>n / 4$, then $H_{L, \psi}^{1}\left(\mathbb{R}^{n}\right)=H_{L, \operatorname{mol}(N)}^{1}\left(\mathbb{R}^{n}\right)$, and when $A$ is self-adjoint, then also $H_{L, \psi}^{1}\left(\mathbb{R}^{n}\right)=H_{L, \text { at }(N)}^{1}\left(\mathbb{R}^{n}\right)$. 
Proof. We will use (3.20) to show that $(\mathrm{H} 4)_{\Psi}$ holds with $\kappa=n$. The hypotheses of Theorem 3.10 will then be satisfied, since it was noted above that $L$ satisfies (H1)-(H3) with $\omega=\omega_{L}$ and $m=2$. To this end, choose $\theta \in\left(\omega_{L}, \pi / 2\right)$, define the nondegenerate function $\tilde{\psi}(z)=z e^{-z}$ on $S_{\theta}^{o} \cup\{0\}$ and note that $\tilde{\psi} \in \Psi^{\beta}\left(S_{\theta}^{o}\right)$ for any $\beta>n / 4$. For each $F \in T_{0}^{2}\left(\mathbb{R}_{+}^{n+1}\right)$, as defined in Remark 3.11, there is a ball $B \subseteq M$ and $r>1$ such that $\operatorname{sppt}(F) \subseteq B \times[1 / r, r]$, and so we have

$$
\begin{aligned}
\left\|\mathcal{S}_{\tilde{\psi}^{*}}^{L^{*}} F\right\|_{q^{\prime}} & =\left\|\int_{1 / r}^{r} t^{2} L^{*} e^{-t^{2} L^{*}} F_{t} \frac{d t}{t}\right\|_{q^{\prime}} \\
& \leq \int_{1 / r}^{r}\left\|e^{-\left(t^{2} / 2\right) L^{*}} t^{2} L^{*} e^{-\left(t^{2} / 2\right) L^{*}} F_{t}\right\|_{q^{\prime}} \frac{d t}{t} \\
& \leq \int_{1 / r}^{r} g\left(t^{2} / 2\right)\left\|t^{2} L^{*} e^{-\left(t^{2} / 2\right) L^{*}} F_{t}\right\|_{2} \frac{d t}{t} \\
& \lesssim\left(\int_{1 / r}^{r}\left(g\left(t^{2} / 2\right)\right)^{2} \frac{d t}{t}\right)^{1 / 2}\left(\int_{0}^{\infty}\left\|F_{t}\right\|_{2}^{2} \frac{d t}{t}\right)^{1 / 2} \\
& \lesssim\|F\|_{T^{2}},
\end{aligned}
$$

where the third line uses (3.20), and the fourth line uses the analyticity of the semigroup $\left(e^{-t L^{*}}\right)_{t>0}$ (see, for instance, Theorem II.4.6 in [19]) followed by the Cauchy-Schwarz inequality. This shows that $\mathcal{S}_{\tilde{\psi}^{*}}^{L^{*}}\left(T_{0}^{2}\left(\mathbb{R}_{+}^{n+1}\right)\right) \subseteq L^{q^{\prime}}\left(\mathbb{R}^{n}\right)$, so Remark 3.11 implies that $(\mathrm{H} 4)_{\Psi}$ holds with $\kappa=n$, as required.

We have now shown that the hypotheses of Theorem 3.10 hold. Moreover, when $q=1$, the hypotheses of Theorem 3.13 follow. The conclusions of those two theorems complete the proof, except for the atomic characterisation in the case when $q=1$ and $A$ is self-adjoint, but then $L=-\operatorname{div} A \nabla$ satisfies the requirements of Theorem 1.3 (see Proposition 3.2 in [5] for a proof of the Davies-Gaffney estimates (5.1)), so we refer the reader to the proof of that theorem in Section 5 .

Theorem 1.2 is a special case of the above result.

Proof of Theorem 1.2. This is a special case of Theorem 3.16, since property (1.4) corresponds to property (3.20) with $q=1$.

\section{Self-adjoint operators with finite propagation speed}

We now restrict the theory of the previous section to the context of any selfadjoint operator $D: \operatorname{Dom}(D) \subseteq L^{2}(\mathcal{V}) \rightarrow L^{2}(\mathcal{V})$ for which the associated unitary $C_{0}$-group $\left(e^{i t D}\right)_{t \in \mathbb{R}}$ has finite propagation speed. The existence of this group is guaranteed by Stone's theorem because $D$ is self-adjoint. The defining features of such a group are that the mapping $t \mapsto e^{i t D}$ is strongly continuous from $\mathbb{R}$ to $\mathcal{L}\left(L^{2}(\mathcal{V})\right)$ with $e^{i(s+t) D}=e^{i s D} e^{i t D},\left.e^{i t D}\right|_{t=0}=I$ and $\left.\frac{d}{d t}\left(e^{i t D} u\right)\right|_{t=0}=i D u$ for all $u \in \operatorname{Dom}(D)=\left\{u \in L^{2}(\mathcal{V}):\left.\frac{d}{d t}\left(e^{i t D} u\right)\right|_{t=0}\right.$ exists in $\left.L^{2}(\mathcal{V})\right\}$. An introduction to the theory of such groups can be found in [24], [19]. The group $\left(e^{i t D}\right)_{t \in \mathbb{R}}$ is said to have finite propagation speed when there exists a finite constant $c_{D}>0$ such 
that for all $u \in L^{2}(\mathcal{V})$ satisfying $\operatorname{sppt}(u) \subseteq F \subseteq M$ and all $t \in \mathbb{R}$, it holds that $\operatorname{sppt}\left(e^{i t D} u\right) \subseteq\left\{x \in M: \rho(\{x\}, F) \leq c_{D}|t|\right\}$. We begin by establishing that these assumptions allow us to apply the theory from the previous section with $\mathcal{D}=D$, $\omega=0$ and $m=1$.

Lemma 4.1. If $D$ is a self-adjoint operator on $L^{2}(\mathcal{V})$ and the group $\left(e^{i t D}\right)_{t \in \mathbb{R}}$ has finite propagation speed $c_{D}>0$, then $D$ satisfies $(\mathrm{H} 1)-(\mathrm{H} 3)$ with $\omega=0$ and $m=1$.

Proof. Since $D$ is self-adjoint, it satisfies (H1) and (H2) with $\omega=0, C_{\theta}=1 / \sin \theta$ and $c_{\theta}=1$. It remains to prove (H3). Let $E$ and $F$ denote measurable subsets of $M$. The finite propagation speed implies that $\mathbf{1}_{E} e^{i t D} \mathbf{1}_{F}=0$ whenever $\rho(E, F)>$ $c_{D}|t|$. For all $z \in \mathbb{C}$ with $\operatorname{Im}( \pm z)>0$, we use the integral representation of the resolvent $(z I-D)^{-1}=\mp i \int_{0}^{\infty} e^{ \pm i z t} e^{\mp i t D} d t$ to obtain

$$
\left\|\mathbf{1}_{E}(z I-D)^{-1} \mathbf{1}_{F}\right\| \leq \int_{\rho(E, F) / c_{D}}^{\infty}\left|e^{ \pm i z t}\right|\left\|\mathbf{1}_{E} e^{\mp i t D} \mathbf{1}_{F}\right\| d t \leq \int_{\rho(E, F) / c_{D}}^{\infty} e^{-(\operatorname{Im}( \pm z)) t} d t .
$$

For each $\theta \in(0, \pi / 2)$, it follows that

$$
\left\|\mathbf{1}_{E}(z I-D)^{-1} \mathbf{1}_{F}\right\| \leq \frac{C_{\theta}}{|z|} \exp \left(-\frac{\rho(E, F)|z|}{c_{D} C_{\theta}}\right) \quad \forall z \in \mathbb{C} \backslash S_{\theta},
$$

which implies (H3) with $m=1$.

The algebra of complex-valued bounded Borel measurable functions on $\mathbb{R}$ is denoted by $B^{\infty}(\mathbb{R})$. The spectral theorem for self-adjoint operators provides $D$ with a bounded $B^{\infty}(\mathbb{R})$ functional calculus such that $\|f(D)\| \leq\|f\|_{\infty}$ for all $f \in$ $B^{\infty}(\mathbb{R})$. This coincides with the holomorphic functional calculus defined by $(3.2)$ and (3.5) when $f \in H^{\infty}\left(S_{\theta}^{o} \cup\{0\}\right)$ because the holomorphic functional calculus is unique with respect to (3.6)-(3.8). In particular, it is well known (see [25], Chapter XX, §1) that the Borel functional calculus is an algebra homomorphism

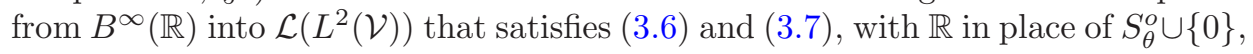
as well as the following convergence lemma, which is related to (3.8):

If $\left(f_{n}\right)_{n}$ is a sequence in $B^{\infty}(\mathbb{R})$ that converges pointwise to a function $f$ in $B^{\infty}(\mathbb{R})$, and $\sup _{n}\left\|f_{n}\right\|_{\infty}<\infty$, then $\lim _{n} f_{n}(D) u=f(D) u$ for all $u \in L^{2}(\mathcal{V})$.

The orthogonal decomposition $L^{2}(\mathcal{V})=\overline{\mathrm{R}(D)} \stackrel{\perp}{\oplus} N(D)$ and the properties of the Borel functional calculus allow us to prove the following Calderón reproducing formula.

Proposition 4.2. Suppose that $D$ is self-adjoint on $L^{2}(\mathcal{V})$. If $f$ and $g$ in $B^{\infty}(\mathbb{R})$ satisfy $f(0) g(0)=0, \int_{0}^{\infty}|f( \pm t) g( \pm t)| \frac{d t}{t}<\infty$ and $\int_{0}^{\infty} f( \pm t) g( \pm t) \frac{d t}{t}=1$, then

$$
\int_{0}^{\infty} f_{t}(D) g_{t}(D) u \frac{d t}{t}=\mathrm{P}_{\overline{R(D)}} u \quad \forall u \in L^{2}(\mathcal{V})
$$

where $\mathrm{P} \overline{R(D)}$ denotes the projection from $L^{2}(\mathcal{V})$ onto $\overline{R(D)}$. 
Proof. Suppose that $f$ and $g$ in $B^{\infty}(\mathbb{R})$ satisfy the hypotheses of the proposition. For each $n \in \mathbb{N}$, we have

$$
h_{n}(x):=\int_{\frac{1}{n}}^{n} f_{t}(x) g_{t}(x) \frac{d t}{t}= \begin{cases}\int_{x / n}^{x n} f(t) g(t) \frac{d t}{t}, & \text { if } x>0 \\ 0, & \text { if } x=0 \\ \int_{|x| / n}^{|x| n} f(-t) g(-t) \frac{d t}{t}, & \text { if } x<0 .\end{cases}
$$

The sequence $\left(h_{n}\right)_{n}$ converges pointwise on $\mathbb{R}$ to the characteristic function $\mathbf{1}_{\mathbb{R} \backslash\{0\}}$, and $\sup _{n}\left\|h_{n}\right\|_{\infty} \leq \int_{0}^{\infty}|f( \pm t) g( \pm t)| \frac{d t}{t}<\infty$, so it follows from (4.1) that

$$
\int_{0}^{\infty} f_{t}(D) g_{t}(D) u \frac{d t}{t}=\lim _{n \rightarrow \infty} h_{n}(D) u=\mathbf{1}_{\mathbb{R} \backslash\{0\}}(D) u=\mathrm{P}_{\overline{\mathrm{R}(D)}} u \quad \forall u \in L^{2}(\mathcal{V}),
$$

where the final equality relies on the fact that $\mathbf{1}_{\mathbb{R}}(D)=I$ and $\mathbf{1}_{\{0\}}(D)=\mathrm{P}_{\mathrm{N}(D)}$.

We now require a class of functions that interact well with finite propagation speed. To this end, a function on $\mathbb{R}$ is called nondegenerate when it is not identically zero on $(0, \infty)$ nor on $(-\infty, 0)$. The Fourier transform of any Schwartz function $f \in \mathcal{S}(\mathbb{R})$ is denoted by $\widehat{f}$. For $\delta>0$ and $N \in \mathbb{N}$, define

$$
\begin{aligned}
\widetilde{\Theta}^{\delta}(\mathbb{R}) & =\{\varphi \in \mathcal{S}(\mathbb{R}): \operatorname{sppt} \widehat{\varphi} \subseteq[-\delta, \delta]\}, \\
\widetilde{\Psi}_{N}^{\delta}(\mathbb{R}) & =\left\{\eta \in \widetilde{\Theta}^{\delta}(\mathbb{R}): \partial^{k-1} \eta(0)=0 \text { for all } k \in\{1, \ldots, N\}\right\},
\end{aligned}
$$

$\widetilde{\Theta}(\mathbb{R})=\bigcup_{\delta>0} \widetilde{\Theta}^{\delta}(\mathbb{R}), \widetilde{\Psi}_{N}(\mathbb{R})=\bigcup_{\delta>0} \widetilde{\Psi}_{N}^{\delta}(\mathbb{R})$ and $\widetilde{\Psi}(\mathbb{R})=\widetilde{\Psi}_{1}(\mathbb{R})$. For $\varphi \in \widetilde{\Theta}(\mathbb{R})$, the Fourier inversion formula and the $B^{\infty}(\mathbb{R})$ functional calculus imply that

$$
\varphi(D) u=\frac{1}{2 \pi} \int_{\mathbb{R}} \widehat{\varphi}(t) e^{i t D} u d t \quad \forall u \in L^{2}(\mathcal{V}) .
$$

For $\eta \in \widetilde{\Psi}(\mathbb{R})$, using the $B^{\infty}(\mathbb{R})$ functional calculus, define $\mathcal{Q}_{\eta}^{D}$ in $\mathcal{L}\left(L^{2}, T^{2}\right)$ by $\left(\mathcal{Q}_{\eta}^{D} u\right)_{t}=\eta(t D) u$, and $\mathcal{S}_{\eta}^{D}$ in $\mathcal{L}\left(T^{2}, L^{2}\right)$ by $\mathcal{S}_{\eta}^{D} U=\int_{0}^{\infty} \eta(s D) U_{s} \frac{d s}{s}$, as well as the space $E_{D, \eta}^{p}(\mathcal{V})=\mathcal{S}_{\eta}^{D}\left(T^{p} \cap T^{2}\right)$. This extends Definitions 3.2 and 3.4, which use the $H^{\infty}\left(S_{\theta}^{o} \cup\{0\}\right)$ functional calculus. Also, note that

$$
\eta(D) u=\mathrm{P}_{\overline{\mathrm{R}(D)}} \eta(D) \mathrm{P}_{\overline{\mathrm{R}(D)}} u \quad \forall u \in L^{2}(\mathcal{V}),
$$

since $\eta(0)=0$ and $\mathbf{1}_{\{0\}}(D)=\mathrm{P}_{\mathrm{N}(D)}$.

The following corollary of Proposition 4.2 extends the Calderón reproducing formula in Proposition 3.3 and allows us to incorporate $\widetilde{\Psi}(\mathbb{R})$ class functions into the theory of Section 3.

Corollary 4.3. Suppose that $D$ is a self-adjoint operator on $L^{2}(\mathcal{V})$. If $\sigma, \tau>0$, $\theta \in(0, \pi / 2)$ and $\eta \in \widetilde{\Psi}(\mathbb{R})$ is nondegenerate, then there exists a nondegenerate $\psi \in \Psi_{\sigma}^{\tau}\left(S_{\theta}^{o}\right)$ such that $\mathcal{S}_{\psi}^{D} \mathcal{Q}_{\eta}^{D} u=\mathcal{S}_{\eta}^{D} \mathcal{Q}_{\psi}^{D} u=\mathrm{P} \overline{R(D)} u$ for all $u \in L^{2}(\mathcal{V})$. 
Proof. Suppose that $\eta \in \widetilde{\Psi}_{N}^{\delta}(\mathbb{R})$ for some $\delta>0$ and $N \in \mathbb{N}$. It follows by the PaleyWiener theorem that $\eta$ extends to an entire function satisfying $|\eta(z)| \leq C e^{\delta|z|}$ for some constant $C>0$ and all $z \in \mathbb{C}$. Now consider $\sigma, \tau>0$ and $\theta \in(0, \pi / 2)$. When $\operatorname{Re}(z)>0$, define $\psi(z)=\alpha_{+} z^{\sigma} e^{-2 \delta z \sec \theta} \eta^{*}(z)$, and when $\operatorname{Re}(z)<0$, define $\psi(z)=\alpha_{-}(-z)^{\sigma} e^{2 \delta z \sec \theta} \eta^{*}(z)$, where $\alpha_{ \pm}$are the normalising constants defined by

$$
\alpha_{+} \int_{0}^{\infty} t^{\sigma} e^{-2 \delta t \sec \theta}|\eta(t)|^{2} \frac{d t}{t}=1 \quad \text { and } \quad \alpha_{-} \int_{0}^{\infty} t^{\sigma} e^{-2 \delta t \sec \theta}|\eta(-t)|^{2} \frac{d t}{t}=1 .
$$

The integrals above are positive, so the normalising constants exist, and for all $z \in \mathbb{C}$ with $\operatorname{Re}(z) \neq 0$, we have

$$
\int_{0}^{\infty} \psi(t z) \eta(t z) \frac{d t}{t}=1
$$

Finally, define $\psi(0)=0$ so that $\psi \in \Psi_{\sigma}^{\tau}\left(S_{\theta}^{o}\right)$, and since $\psi$ is clearly nondegenerate, the result follows from Proposition 4.2.

The next result shows how $\widetilde{\Theta}(\mathbb{R})$ functions interact with finite propagation speed. In particular, the off-diagonal estimate in (4.5) is much sharper than that in $(3.9)$.

Lemma 4.4. Suppose that $D$ is a self-adjoint operator on $L^{2}(\mathcal{V})$ and the group $\left(e^{i t D}\right)_{t \in \mathbb{R}}$ has finite propagation speed $c_{D}>0$. If $\delta>0$ and $\varphi \in \widetilde{\Theta}^{\delta}(\mathbb{R})$, then

$$
\left\|\mathbf{1}_{E} \varphi_{t}(D) \mathbf{1}_{F}\right\| \leq \frac{1}{\pi}\|\widehat{\varphi}\|_{\infty} \max \left\{\delta-\frac{\rho(E, F)}{c_{D} t}, 0\right\} \leq C e^{-\rho(E, F) / t}
$$

for all $t>0$, all measurable sets $E, F \subseteq M$, and some $C>0$.

Proof. Suppose that $\varphi \in \widetilde{\Theta}(\mathbb{R})$ with sppt $\widehat{\varphi} \subseteq[-\delta, \delta]$. It follows from (4.3) that

$$
\varphi_{t}(D) u=\frac{1}{2 \pi} \int_{-\infty}^{\infty} \widehat{\varphi_{t}}(s) e^{i s D} u d s=\frac{1}{2 \pi} \int_{|s| \leq \delta t} \widehat{\varphi}\left(\frac{s}{t}\right) e^{i s D} u \frac{d s}{t} \forall t>0, \forall u \in L^{2}(\mathcal{V}) .
$$

Suppose that $E$ and $F$ are measurable subsets of $M$. The finite propagation speed implies $\mathbf{1}_{E} e^{i s D} \mathbf{1}_{F}=0$ whenever $\rho(E, F)>c_{D}|s|$, hence $\mathbf{1}_{E} \varphi_{t}(D) \mathbf{1}_{F}=0$ whenever $\rho(E, F) / c_{D}>\delta t$ by the preceding formula. In addition, if $\rho(E, F) / c_{D} \leq \delta t$, then

$$
\begin{aligned}
\left\|\mathbf{1}_{E} \varphi_{t}(D) \mathbf{1}_{F} u\right\|_{2} & \leq \frac{1}{2 \pi} \int_{\rho(E, F) / c_{D} \leq|s| \leq \delta t}\left|\widehat{\varphi}\left(\frac{s}{t}\right)\right|\left\|\mathbf{1}_{E} e^{i s D} \mathbf{1}_{F} u\right\|_{2} \frac{d s}{t} \\
& \leq \frac{1}{2 \pi} \int_{\rho(E, F) / c_{D} t \leq|\sigma| \leq \delta}|\widehat{\varphi}(\sigma)| d \sigma\|u\|_{2} \\
& \leq \frac{1}{\pi}\|\widehat{\varphi}\|_{\infty}\left(\delta-\frac{\rho(E, F)}{c_{D} t}\right)\|u\|_{2} \leq \frac{1}{\pi}\|\widehat{\varphi}\|_{\infty} \delta e^{\delta c_{D}} e^{-\rho(E, F) / t}\|u\|_{2}
\end{aligned}
$$

for all $u \in L^{2}(\mathcal{V})$, which completes the proof. 
The next two results show that $E_{D, \psi}^{p}(\mathcal{V})=E_{D, \eta}^{p}(\mathcal{V})$ for suitable $\psi$ in $\Psi\left(S_{\theta}^{o}\right)$ and $\eta$ in $\widetilde{\Psi}(\mathbb{R})$. The results rely on some technical off-diagonal estimates that we postpone until Section 6. The first result is an extension of Theorem 4.9 in [9].

Proposition 4.5. Suppose that $M$ is a doubling metric measure space satisfying $\left(\mathrm{D}_{\kappa}\right)$, that $D$ is a self-adjoint operator on $L^{2}(\mathcal{V})$, and the group $\left(e^{i t D}\right)_{t \in \mathbb{R}}$ has finite propagation speed. If $p \in[1,2], \theta \in(0, \pi / 2), N \in \mathbb{N}, N>\kappa / 2$, $\psi \in \Psi_{2 N+1}^{N+1}\left(S_{\theta}^{o}\right), \eta \in \widetilde{\Psi}_{N}(\mathbb{R})$ and $\tilde{\eta} \in \widetilde{\Psi}(\mathbb{R})$, then

$$
\left\|\mathcal{Q}_{\tilde{\eta}}^{D} \mathcal{S}_{\psi}^{D} U\right\|_{T^{p}} \lesssim\|U\|_{T^{p}} \quad \text { and } \quad\left\|\mathcal{Q}_{\psi}^{D} \mathcal{S}_{\eta}^{D} U\right\|_{T^{p}} \lesssim\|U\|_{T^{p}}
$$

for all $U \in T^{p} \cap T^{2}$.

Proof. The proof follows Theorem 4.9 in [9]. When $p=2$, the result is immediate. When $p=1$, it suffices to show that there exists $C>0$ such that

$$
\left\|\mathcal{Q}_{\tilde{\eta}}^{D} \mathcal{S}_{\psi}^{D}(A)\right\|_{T^{1}} \leq C \quad \text { and } \quad\left\|\mathcal{Q}_{\psi}^{D} \mathcal{S}_{\eta}^{D}(A)\right\|_{T^{1}} \leq C
$$

for all $A$ that are $T^{1}$-atoms, since Theorem 3.1 applies. When $p \in(1,2)$, the result then follows by the interpolation in (3.11). Therefore, it remains to prove (4.6).

Lemma 6.3 applied with $(m, n, N, \sigma, \tau, \delta)=(1, N, 1,2 N+1, N+1,1)$ shows that

$$
\left\|\mathbf{1}_{E}\left(\tilde{\eta}_{t} \psi_{s}\right)(D) \mathbf{1}_{F}\right\| \leq C \begin{cases}(s / t)^{N}\langle t / \rho(E, F)\rangle^{N}, & \text { if } 0<s \leq t \\ (t / s)\langle s / \rho(E, F)\rangle^{2 N+1}, & \text { if } 0<t \leq s\end{cases}
$$

for all measurable sets $E, F \subseteq M$. Since $\left(\psi_{t} \eta_{s}\right)(D)=\left(\eta_{s} \psi_{t}\right)(D)$, Lemma 6.3 applied with $(m, n, N, \sigma, \tau, \delta)=(N, 1, N, 2 N+1, N+1,1)$ also shows that

$$
\left\|\mathbf{1}_{E}\left(\psi_{t} \eta_{s}\right)(D) \mathbf{1}_{F}\right\| \leq C \begin{cases}(s / t)^{N}\langle t / \rho(E, F)\rangle^{3 N}, & \text { if } 0<s \leq t \\ (t / s)\langle s / \rho(E, F)\rangle^{2 N-1}, & \text { if } 0<t \leq s\end{cases}
$$

for all measurable sets $E, F \subseteq M$. These estimates combined with $\left(\mathrm{D}_{\kappa}\right)$ prove (4.6) as in Step 2 of the proof of Theorem 4.9 in [9]. This completes the proof.

The second result is an extension of Lemma 5.2 in [9].

Proposition 4.6. Suppose that $M$ is a doubling metric measure space satisfying $\left(\mathrm{D}_{\kappa}\right)$, that $D$ is a self-adjoint operator on $L^{2}(\mathcal{V})$, and the group $\left(e^{i t D}\right)_{t \in \mathbb{R}}$ has finite propagation speed. If $p \in[1,2], \theta \in(0, \pi / 2), \beta>\kappa / 2, N \in \mathbb{N}, N>\kappa / 2$, and all of $\psi \in \Psi_{\beta}\left(S_{\theta}^{o}\right), \eta \in \widetilde{\Psi}_{N}(\mathbb{R})$ and $\tilde{\eta} \in \widetilde{\Psi}(\mathbb{R})$ are nondegenerate, then

$$
\mathcal{S}_{\psi}^{D}\left(T^{p} \cap T^{2}\right)=\mathcal{S}_{\eta}^{D}\left(T^{p} \cap T^{2}\right)=\left\{u \in \overline{R(D)}: \mathcal{Q}_{\tilde{\eta}}^{D} u \in T^{p}\right\}
$$

with the norm equivalence

$$
\|u\|_{E_{D, \psi}^{p}} \approx\|u\|_{E_{D, \eta}^{p}} \approx\left\|\mathcal{Q}_{\tilde{\eta}}^{D} u\right\|_{T^{p}} \quad \forall u \in E_{D, \psi}^{p}=\mathcal{S}_{\psi}^{D}\left(T^{p} \cap T^{2}\right) .
$$

If, in addition, the completion $H_{D, \psi}^{p}$ of $E_{D, \psi}^{p}$ in $L^{p}$ exists, then there are unique extensions $\widetilde{\mathcal{S}}_{\eta}^{D} \in \mathcal{L}\left(T^{p}, H_{D, \psi}^{p}\right)$ and $\widetilde{\mathcal{Q}}_{\tilde{\eta}}^{D} \in \mathcal{L}\left(H_{D, \psi}^{p}, T^{p}\right)$ such that $\widetilde{\mathcal{S}}_{\eta}^{D}=\mathcal{S}_{\eta}^{D}$ on $T^{p} \cap T^{2}$ 
and $\widetilde{\mathcal{Q}}_{\tilde{\eta}}^{D}=\mathcal{Q}_{\tilde{\eta}}^{D}$ on $E_{D, \psi}^{p}$. It also holds that $H_{D, \psi}^{p}=\widetilde{\mathcal{S}}_{\eta}^{D}\left(T^{p}\right)$ with the norm equivalence

$$
\|u\|_{H_{D, \psi}^{p}} \bar{\sim} \inf \left\{\|U\|_{T^{p}}: U \in T^{p} \text { and } u=\widetilde{\mathcal{S}}_{\eta}^{D} U\right\} \approx\left\|\widetilde{\mathcal{Q}}_{\tilde{\eta}}^{D} u\right\|_{T^{p}} \quad \forall u \in H_{D, \psi}^{p} .
$$

Moreover, if $E_{D, \psi}^{p}$ is dense in $H_{D, \psi}^{p} \cap L^{2}$, then also $\widetilde{\mathcal{Q}}_{\tilde{\eta}}^{D}=\mathcal{Q}_{\tilde{\eta}}^{D}$ on $H_{D, \psi}^{p} \cap L^{2}$.

Proof. It suffices, by Theorem 3.6, to prove the result for a fixed nondegenerate $\psi$ in $\Psi_{\beta}\left(S_{\theta}^{o}\right)$, so we select $\psi$ in $\Psi_{2 N+1}^{N+1}\left(S_{\theta}^{o}\right)$ satisfying $\int_{0}^{\infty} \psi( \pm t)^{2} \frac{d t}{t}=1$. Suppose that both $\eta \in \widetilde{\Psi}_{N}(\mathbb{R})$ and $\tilde{\eta} \in \widetilde{\Psi}(\mathbb{R})$ are nondegenerate, and then use Corollary 4.3 to obtain $\varphi$ and $\tilde{\varphi}$ in $\Psi_{2 N+1}^{N+1}\left(S_{\theta}^{o}\right)$ such that $\mathcal{S}_{\eta}^{D} \mathcal{Q}_{\varphi}^{D}=\mathcal{S}_{\tilde{\varphi}}^{D} \mathcal{Q}_{\tilde{\eta}}^{D}=\mathrm{P} \overline{\mathrm{R}(D)}=\mathcal{S}_{\psi}^{D} \mathcal{Q}_{\psi}^{D}$. The proof of (4.7) and (4.8) proceeds in three parts corresponding to the set inclusions

$$
\mathcal{S}_{\psi}^{D}\left(T^{p} \cap T^{2}\right) \stackrel{(\mathrm{i})}{\subseteq} \mathcal{S}_{\eta}^{D}\left(T^{p} \cap T^{2}\right) \stackrel{(\mathrm{ii})}{\subseteq}\left\{u \in \overline{\mathrm{R}(D)}: \mathcal{Q}_{\tilde{\eta}}^{D} u \in T^{p}\right\} \stackrel{(\mathrm{iii})}{\subseteq} \mathcal{S}_{\psi}^{D}\left(T^{p} \cap T^{2}\right)
$$

and the related norm estimates.

(i) If $u \in \mathcal{S}_{\psi}^{D}\left(T^{p} \cap T^{2}\right)$, then (3.14) implies that $u \in \overline{\mathrm{R}(D)}$ and $\mathcal{Q}_{\psi}^{D} u \in T^{p} \cap T^{2}$, so $u=\mathcal{S}_{\eta}^{D}\left(\mathcal{Q}_{\varphi}^{D} \mathcal{S}_{\psi}^{D} \mathcal{Q}_{\psi}^{D} u\right)$ and (3.13) followed by (3.15) imply that

$$
\|u\|_{E_{D, \eta}^{p}} \leq\left\|\mathcal{Q}_{\varphi}^{D} \mathcal{S}_{\psi}^{D}\left(\mathcal{Q}_{\psi}^{D} u\right)\right\|_{T^{p}} \lesssim\left\|\mathcal{Q}_{\psi}^{D} u\right\|_{T^{p}} \approx\|u\|_{E_{D, \psi}^{p}} .
$$

(ii) If $u \in \mathcal{S}_{\eta}^{D}\left(T^{p} \cap T^{2}\right)$, then $u \in \overline{\mathrm{R}(D)}$ by (4.4), and there exists $V \in T^{p} \cap T^{2}$ such that $u=\mathcal{S}_{\eta}^{D}(V)$ and $\|V\|_{T^{p}} \leq 2\|u\|_{E_{D, \eta}^{p}}$, so by applying Proposition 4.5 twice we obtain

$$
\left\|\mathcal{Q}_{\tilde{\eta}}^{D} u\right\|_{T^{p}}=\left\|\mathcal{Q}_{\tilde{\eta}}^{D} \mathcal{S}_{\psi}^{D}\left(\mathcal{Q}_{\psi}^{D} \mathcal{S}_{\eta}^{D} V\right)\right\|_{T^{p}} \lesssim\|V\|_{T^{p}} \lesssim\|u\|_{E_{D, \eta}^{p}}
$$

(iii) If $u \in \overline{\mathrm{R}(D)}$ and $\mathcal{Q}_{\tilde{\eta}}^{D} u \in T^{p}$, then $u=\mathcal{S}_{\psi}^{D}\left(\mathcal{Q}_{\psi}^{D} \mathcal{S}_{\tilde{\varphi}}^{D} \mathcal{Q}_{\tilde{\eta}}^{D} u\right)$, so (3.13) implies that

$$
\|u\|_{E_{D, \psi}^{p}} \leq\left\|\mathcal{Q}_{\psi}^{D} \mathcal{S}_{\tilde{\varphi}}^{D}\left(\mathcal{Q}_{\tilde{\eta}}^{D} u\right)\right\|_{T^{p}} \lesssim\left\|\mathcal{Q}_{\tilde{\eta}}^{D} u\right\|_{T^{p}}
$$

We obtain (4.9) and the related properties by the arguments used to prove (3.16) and the related properties. This completes the proof.

We now introduce hypothesis $(\mathrm{H} 4)_{\widetilde{\Psi}}$ on $D$ in order to prove that the completion of $E_{D, \psi}^{p}(\mathcal{V})$ in $L^{p}(\mathcal{V})$ exists. This provides an alternative to hypothesis $(\mathrm{H} 4)_{\Psi}$ from Theorem 3.10 when $D$ is self-adjoint and $\left(e^{i t D}\right)_{t \in \mathbb{R}}$ has finite propagation speed. The advantage of hypothesis $(\mathrm{H} 4)_{\widetilde{\Psi}}$ is that $\mathcal{S}_{\eta}^{D} F$ has compact support whenever $F$ has compact support, and as such, it is more easily verified that $\mathcal{S}_{\eta}^{D} F \in L^{q^{\prime}}(\mathcal{V})$.

Theorem 4.7. Suppose that $M$ is a doubling metric measure space satisfying $\left(\mathrm{D}_{\kappa}\right)$, that $D$ is a self-adjoint operator on $L^{2}(\mathcal{V})$, and $\left(e^{i t D}\right)_{t \in \mathbb{R}}$ has finite propagation speed. If $1 \leq q \leq p \leq 2, \theta \in(0, \pi / 2), \beta>\kappa / 2, \psi \in \Psi_{\beta}\left(S_{\theta}^{o}\right)$ is nondegenerate and

(H4) there exists a nondegenerate function $\eta \in \widetilde{\Psi}(\mathbb{R})$ such that the set $\left\{F \in T^{2} \cap T^{q^{\prime}}: \mathcal{S}_{\eta}^{D} F \in L^{q^{\prime}}(\mathcal{V})\right\}$ is weak-star dense in $T^{p^{\prime}}\left(\mathcal{V}_{+}\right)$,

where $1 / q+1 / q^{\prime}=1$, then the completion $H_{D, \psi}^{p}(\mathcal{V})$ of $E_{D, \psi}^{p}(\mathcal{V})$ in $L^{p}(\mathcal{V})$ exists. Moreover, it holds that $H_{D, \psi}^{p}(\mathcal{V}) \cap L^{2}(\mathcal{V})=E_{D, \psi}^{p}(\mathcal{V})$. 
Proof. Following the proof of Theorem 3.10, let $\left(u_{n}\right)_{n}$ denote a Cauchy sequence in $E_{D, \psi}^{p}$ that converges to 0 in $L^{p}$. We need to show that $\left(u_{n}\right)_{n}$ converges to 0 in $E_{D, \psi}^{p}$. To see this, fix $\eta$ in $\widetilde{\Psi}(\mathbb{R})$ satisfying $(\mathrm{H} 4)_{\widetilde{\Psi}}$. For all $n \in \mathbb{N}$, we have by (4.8) that

$$
\left\|u_{n}\right\|_{E_{D, \psi}^{p}} \approx\left\|\mathcal{Q}_{\eta}^{D} u_{n}\right\|_{T^{p}}
$$

We conclude by repeating the proof of Theorem 3.10 with (3.18) replaced by (4.10) and $\mathcal{Q}_{\tilde{\psi}}^{\mathcal{D}}$ replaced by $\mathcal{Q}_{\eta}^{D}$.

Remark 4.8. In the context of Theorem 4.7, when $M$ is a complete Riemannian manifold, hypothesis $(\mathrm{H} 4)_{\widetilde{\Psi}}$ holds whenever $\mathcal{S}_{\eta}^{D}\left(C_{c}^{\infty}\left(\mathcal{V}_{+}\right)\right) \subseteq L^{q^{\prime}}(\mathcal{V})$, where $C_{c}^{\infty}\left(\mathcal{V}_{+}\right)$denotes the space of smooth compactly supported sections in $T^{2}\left(\mathcal{V}_{+}\right)$. This is because $C_{c}^{\infty}\left(\mathcal{V}_{+}\right)$is weak-star dense in $T^{p^{\prime}}\left(\mathcal{V}_{+}\right)$for all $p \in[1,2]$. To see this, a mollification argument can be applied in combination with Remark 3.11.

\subsection{Atomic theory}

We obtain a characterisation of $H_{D, \psi}^{1}(\mathcal{V})$ in terms of the atoms from Definition 3.8 and the space $H_{D, \text { at }(N)}^{1}(\mathcal{V})$ from Definition 3.12 .

Theorem 4.9. Suppose that $M$ is a doubling metric measure space satisfying $\left(\mathrm{D}_{\kappa}\right)$, that $D$ is a self-adjoint operator on $L^{2}(\mathcal{V})$, and $\left(e^{i t D}\right)_{t \in \mathbb{R}}$ has finite propagation speed. Also, assume that for some $\theta \in(0, \pi / 2), \beta>\kappa / 2$ and nondegenerate $\psi \in \Psi_{\beta}\left(S_{\theta}^{o}\right)$, the completion $H_{D, \psi}^{1}(\mathcal{V})$ of $E_{D, \psi}^{1}(\mathcal{V})$ in $L^{1}(\mathcal{V})$ exists. It follows that if $N \in \mathbb{N}$ and $N>\kappa / 2$, then $H_{D, \psi}^{1}(\mathcal{V})=H_{D, \operatorname{mol}(N)}^{1}(\mathcal{V})=H_{D, \text { at }(N)}^{1}(\mathcal{V})$.

Proof. Suppose that $N \in \mathbb{N}$ and $N>\kappa / 2$. Theorem 3.13 and Lemma 4.1 show that $H_{D, \psi}^{1}=H_{D, \operatorname{mol}(N)}^{1} \supseteq H_{D, \text { at }(N)}^{1}$. It remains to prove that $H_{D, \psi}^{1} \subseteq H_{D, \text { at }(N)}^{1}$. To do this, fix a nondegenerate $\eta$ in $\widetilde{\Psi}_{N}(\mathbb{R})$. We claim that there exists $c>0$ such that $c \mathcal{S}_{\eta}^{D} A$ is an $H_{D}^{1}$-atom of type $N$ whenever $A$ is a $T^{1}$-atom. The claim allows us to prove that $H_{D, \psi}^{1} \subseteq H_{D \text {, at }(N)}^{1}$ by repeating the proof of Theorem 3.13 with $\tilde{\psi}$ replaced by $\eta$ and then relying on (4.8) and (4.9) instead of (3.15) and (3.16).

To prove the claim, let $A$ denote a $T^{1}$-atom and let $B$ denote a ball in $M$ with radius $r(B)>0$ such that $A$ is supported in the tent $T(B)$ and $\|A\|_{T^{2}} \leq \mu(B)^{-1 / 2}$. Note that $A_{t}$ is supported in $B$ when $t \in(0, r(B)]$, and that $\eta_{t}(D) A_{t}=0$ when $t>r(B)$. The finite propagation speed, in particular (4.5), then implies that there exists $\alpha>0$, which only depends on $\eta$ and $D$, such that $\eta_{t}(D) A_{t}$ is supported in $\alpha B$ for all $t>0$, hence $\mathcal{S}_{\eta}^{D} A$ is supported in $\alpha B$.

Now set $\tilde{\eta}(x)=x^{-N} \eta(x)$ for all $x \in \mathbb{R} \backslash\{0\}$, and $\tilde{\eta}(0)=\partial^{N} \eta(0) / N$ !, which equals $\lim _{x \rightarrow 0} x^{-N} \eta(x)$. Lemma 6.1 shows that $\tilde{\eta} \in \widetilde{\Theta}(\mathbb{R})$, and so the properties of the $B^{\infty}(\mathbb{R})$ functional calculus imply that the putative atom $a:=\mathcal{S}_{\eta}^{D} A$ has the form

$$
a=\mathcal{S}_{\eta}^{D} A=D^{N}\left(\int_{0}^{\infty} t^{N} \tilde{\eta}_{t}(D) A_{t} \frac{d t}{t}\right)=: D^{N} b .
$$


It remains to verify that $a$ and $b$ above satisfy the atomic bounds in Definition 3.8. We use the doubling property to obtain

$$
\|a\|_{2}=\left\|\mathcal{S}_{\eta}^{D} A\right\|_{2} \lesssim\|A\|_{T^{2}} \leq \mu(B)^{-1 / 2} \lesssim \mu(\alpha B)^{-1 / 2},
$$

and since $A_{t}=0$ for all $t>r(B)$, we also have

$$
\|b\|_{2}=\left\|\mathcal{S}_{\tilde{\eta}}^{D}\left(t^{N} A_{t}\right)\right\|_{2} \lesssim r(B)^{N}\|A\|_{T^{2}} \lesssim(\alpha r(B))^{N} \mu(\alpha B)^{-1 / 2} .
$$

Therefore, there exists $c>0$, which does not depend on $A$, such that $c \mathcal{S}_{\eta}^{D}(A)$ is an $H_{D}^{1}$-atom of type $N$. This proves the claim and completes the proof.

Remark 4.10. The proof of Theorem 4.9 shows that the same result holds when the $L^{1}(\mathcal{V})$ convergence required in Definition 3.12 is replaced with $H_{D, \psi}^{1}(\mathcal{V})$ convergence.

\subsection{The embedding $H_{D}^{p} \subseteq L^{p}$ for smooth differential operators}

We now consider the case when $M$ is a complete Riemannian manifold, which is assumed to be smooth (infinitely differentiable) and connected, with geodesic distance $\rho$ and Riemannian measure $\mu$. The vector bundle $\mathcal{V}$ is also assumed to be smooth, which means that the complex vector bundle $\pi: \mathcal{V} \rightarrow M$ is equipped with a Hermitian metric $\langle\cdot, \cdot\rangle_{x}$ that is infinitely differentiable with respect to $x \in M$. Let $\operatorname{dim}(M)$ denote the dimension of $M$ and let $\operatorname{dim}(\mathcal{V})$ denote the fibre dimension of $\mathcal{V}$. We prove a general result for a class of first-order differential operators on $L^{2}(\mathcal{V})$. The results for the Hodge-Dirac operator in Theorem 1.1 are deduced afterwards.

A smooth-coefficient, first-order, differential operator $D_{c}$ is a linear operator on $L^{2}(\mathcal{V})$ with domain $\operatorname{Dom}\left(D_{c}\right)=C_{c}^{\infty}(\mathcal{V})$ such that on any coordinate patch over which $\mathcal{V}$ is trivial, there are smooth, matrix-valued $\left(\mathcal{L}\left(\mathbb{C}^{\operatorname{dim}(\mathcal{V})}\right)\right.$-valued $)$ functions $\left(A_{j}\right)_{j=0, \ldots, \operatorname{dim}(M)}$ such that the action of $D_{c}$ on that coordinate patch is given by the Euclidean operator $\sum_{j=1}^{\operatorname{dim}(M)} A_{j} \partial_{j}+A_{0}$. For each $x \in M$ in such a coordinate patch and each $\xi \in T_{x}^{*} M$ given by $\xi=\sum_{j=1}^{\operatorname{dim}(M)} \xi_{j} d x^{j}$, the principal symbol $\sigma_{D_{c}}(x, \xi)$ is the endomorphism on the fibre $\mathcal{V}_{x}$ given by $\sum_{j=1}^{\operatorname{dim}(M)} A_{j} \xi_{j}$. A full account of these standard facts, including a coordinate-free definition of the principal symbol, is in [32], Chapter IV, Section 2. Moreover, for any $\eta \in C_{c}^{\infty}(M)$, the principal symbol is given by the commutator $\left[D_{c}, \eta I\right] u=D_{c}(\eta u)-\eta D_{c} u$, since

$$
\left(\sigma_{D_{c}}(x, d \eta(x))\right)(u(x))=\left(\left[D_{c}, \eta I\right] u\right)(x) \quad \forall x \in M, \forall u \in C_{c}^{\infty}(\mathcal{V}),
$$

where $d$ is the exterior derivative.

An operator $D_{c}$ is called symmetric when $\left\langle D_{c} u, v\right\rangle=\left\langle u, D_{c} v\right\rangle$ for all $u, v \in C_{c}^{\infty}(\mathcal{V})$. A symmetric first-order operator has a skew-symmetric principal symbol. Chernoff proved in [13] that if the principal symbol of a symmetric, smooth-coefficient, firstorder, differential operator satisfies a certain bound, then the operator is essentially self-adjoint and generates a group with finite propagation speed (related results are discussed in Remark 4.12). This allows us to prove the following result. 
Theorem 4.11. Suppose that $M$ is a complete Riemannian manifold satisfying $\left(\mathrm{D}_{\kappa}\right)$ and that $\mathcal{V}$ is a smooth vector bundle over $M$. Let $D$ denote the unique self-adjoint extension of a symmetric, smooth-coefficient, first-order, differential operator $D_{c}$ on $L^{2}(\mathcal{V})$ for which there exists $c_{D}>0$ such that the principal symbol $\sigma_{D_{c}}$ satisfies

$$
\left\|\sigma_{D_{c}}(x, \xi)\right\|_{\mathcal{L}\left(\mathcal{V}_{x}\right)} \leq c_{D}|\xi|_{T_{x}^{*} M} \quad \forall x \in M, \forall \xi \in T_{x}^{*} M .
$$

If $p \in[1,2], \theta \in(0, \pi / 2), \beta>\kappa / 2$ and $\psi \in \Psi_{\beta}\left(S_{\theta}^{o}\right)$ is nondegenerate, then the completion $H_{D, \psi}^{p}(\mathcal{V})$ of $E_{D, \psi}^{p}(\mathcal{V})$ in $L^{p}(\mathcal{V})$ exists and $H_{D, \psi}^{p}(\mathcal{V}) \cap L^{2}(\mathcal{V})=E_{D, \psi}^{p}(\mathcal{V})$. Moreover, if $N \in \mathbb{N}$ and $N>\kappa / 2$, then $H_{D, \psi}^{1}(\mathcal{V})=H_{D, \operatorname{mol}(N)}^{1}(\mathcal{V})=H_{D, \text { at }(N)}^{1}(\mathcal{V})$.

Proof. Assumption (4.11) implies that $D_{c}$ is essentially self-adjoint on $L^{2}(\mathcal{V})$ by the result of Chernoff in [13], Theorem 2.2. The results in [13], Theorem 1.3 and Corollary 1.4, also show that the group $\left(e^{i t D}\right)_{t \in \mathbb{R}}$ has finite propagation speed $c_{D}$. Therefore, by Theorems 4.7 and 4.9 , it suffices to prove that $(\mathrm{H} 4)_{\widetilde{\Psi}}$ holds with $q=1$.

First, we require a known estimate for the Sobolev spaces $W^{k, 2}(\mathcal{V})$, where $k \in \mathbb{N}$. If $k>1+\operatorname{dim}(M) / 2$ and $\mathcal{B}$ is a ball in $M$, then there exists $C_{\mathcal{B}}>0$ such that, for all $u \in W^{k, 2}(\mathcal{V})$ with $\operatorname{sppt}(u) \subset \mathcal{B}$, then

$$
\|u\|_{\infty} \leq C_{\mathcal{B}}\|u\|_{W^{k, 2}(\mathcal{V})} .
$$

This Sobolev embedding theorem can be found in [32], Chapter IV, Proposition 1.1.

Second, we require a known energy estimate. If $k \in \mathbb{N}, T>0$, and $\mathcal{B}$ is a ball in $M$, then there exists $C_{T, \mathcal{B}}>0$ such that, for all $u \in C_{c}^{\infty}(\mathcal{V})$ with $\operatorname{sppt}(u) \subset \mathcal{B}$, then

$$
\left\|e^{i t D} u\right\|_{W^{k, 2}(\mathcal{V})} \leq C_{T, \mathcal{B}}\|u\|_{W^{k, 2}(\mathcal{V})} \quad \forall t \in[-T, T] .
$$

This can be proved by the methods in [31], Chapter IV, Section 2, since $v(t)=$ $e^{i t D} u$ solves the initial value problem $d v / d t=i D v$ with $v(0)=u$.

Now choose a nondegenerate $\eta$ in $\widetilde{\Psi}(\mathbb{R})$ and $\delta>0$ such that sppt $\widehat{\eta} \subseteq[-\delta, \delta]$. Fix $k \in \mathbb{N}$ such that $k>1+\operatorname{dim}(M) / 2$ and set $\alpha=1+c_{D} \delta$. For each $F \in C_{c}^{\infty}\left(\mathcal{V}_{+}\right)$, there is a ball $B \subseteq M$ and $r>1$ such that $\operatorname{sppt}(F) \subseteq B \times[1 / r, r]$. It follows that $\operatorname{sppt}\left(e^{i s t D} F_{t}\right) \subseteq\left(1+c_{D}|s| t / r\right) B \subseteq \alpha B$ for all $s \in[-\delta, \delta]$ and $t \in[1 / r, r]$. Hence

$$
\begin{aligned}
\left\|\mathcal{S}_{\eta}^{D} F\right\|_{\infty} & =\left\|\int_{1 / r}^{r}\left(\frac{1}{2 \pi} \int_{-\delta}^{\delta} \widehat{\eta}(s) e^{i s t D} F_{t} d s\right) \frac{d t}{t}\right\|_{\infty} \\
& \lesssim \int_{1 / r}^{r} \int_{-\delta}^{\delta}\left\|e^{i s t D} F_{t}\right\|_{\infty} d s d t \\
& \lesssim \int_{1 / r}^{r} \int_{-\delta}^{\delta}\left\|e^{i s t D} F_{t}\right\|_{W^{k, 2}(\mathcal{V})} d s d t \\
& \lesssim \int_{1 / r}^{r}\left\|F_{t}\right\|_{W^{k, 2}(\mathcal{V})} d t \\
& <\infty
\end{aligned}
$$


where the first line uses (4.3), the third line uses (4.12) with $\mathcal{B}=\alpha B$, the fourth line uses (4.13) with $\mathcal{B}=B$, and the fifth line uses the continuity of $F$ (recall that $\left.F \in C_{c}^{\infty}\left(\mathcal{V}_{+}\right)\right)$. This shows that $\mathcal{S}_{\eta}^{D}\left(C_{c}^{\infty}\left(\mathcal{V}_{+}\right)\right) \subseteq L^{\infty}(\mathcal{V})$, so Remark 4.8 implies that $(\mathrm{H} 4)_{\widetilde{\Psi}}$ holds with $q=1$. This completes the proof.

Remark 4.12. McIntosh and Morris ([27], Theorem 1.1) proved recently that any $C_{0}$-group $\left(e^{i t D}\right)_{t \in \mathbb{R}}$ generated by a first-order system $D$ satisfying (4.11) has finite propagation speed. In particular, finite propagation speed for such groups is not restricted to smooth-coefficient nor self-adjoint systems.

We now prove Theorem 1.1, which fills a gap in the theory of Hardy spaces of differential forms developed by Auscher, McIntosh and Russ [9].

Proof of Theorem 1.1. Let $M$ denote a doubling, complete Riemannian manifold. The bundle $\wedge T^{*} M=\oplus_{k=0}^{\operatorname{dim}(M)} \wedge^{k} T^{*} M$, where $\wedge^{k} T^{*} M$ denotes the $k$ th exterior power of the cotangent bundle $T^{*} M$, is defined with the Hermitian metric induced by the Riemannian metric. The Hodge-Dirac operator $D=d+d^{*}$ is defined initially on $C_{c}^{\infty}\left(\wedge T^{*} M\right)$, where $d$ and $d^{*}$ denote the exterior derivative and its adjoint. This is a symmetric, smooth-coefficient, first-order, differential operator on $L^{2}\left(\wedge T^{*} M\right)$ with principal symbol

$$
\left.\sigma_{D}(x, \xi) \zeta=\xi \wedge \zeta-\xi\right\lrcorner \zeta \quad \forall x \in M, \forall \xi \in T_{x}^{*} M, \forall \zeta \in \wedge T_{x}^{*} M,
$$

where $\wedge$ and $\lrcorner$ denote the exterior and (left) interior products on $\wedge T_{x}^{*} M$. These properties of the Hodge-Dirac operator are well known, and in particular, we have

$$
\left|\sigma_{D}(x, \xi) \zeta\right|_{\wedge T_{x}^{*} M}=|\xi|_{T_{x}^{*} M}|\zeta|_{\wedge T_{x}^{*} M} \quad \forall x \in M, \forall \xi \in T_{x}^{*} M, \forall \zeta \in \wedge T_{x}^{*} M,
$$

so the hypotheses of Theorem 4.11 hold, and its conclusions imply Theorem 1.1.

\section{The embedding $H_{L}^{p} \subseteq L^{p}$ for nonnegative self-adjoint op- erators}

We now combine the theory of the previous two sections to prove Theorem 1.3. The atomic characterisation in Theorem 1.2 is then an immediate corollary. A new proof of Theorem 1.2 for smooth coefficient operators is also presented.

We return to the context of a vector bundle $\mathcal{V}$ over a doubling metric measure space $M$. A nonnegative self-adjoint operator $L: \operatorname{Dom}(L) \subseteq L^{2}(\mathcal{V}) \rightarrow L^{2}(\mathcal{V})$ is said to satisfy Davies-Gaffney estimates when there exist constants $C, c>0$ such that

$$
\left\|\mathbf{1}_{E} e^{-t L} \mathbf{1}_{F} u\right\|_{2} \leq C e^{-c \rho(E, F)^{2} / t}\|u\|_{2}
$$

for all $t>0$, all $u \in L^{2}(\mathcal{V})$ and all measurable sets $E, F \subseteq M$, where $\left(e^{-t L}\right)_{t>0}$ is the analytic semigroup generated by $-L$. The following builds on the theory of Hardy spaces developed for such operators by Hofmann, Lu, Mitrea, Mitrea and Yan $[20]$. 
Proof of Theorem 1.3. Since $L$ is self-adjoint, it satisfies (H1) and (H2) with $\omega=0$, $C_{\theta}=1 / \sin \theta$ and $c_{\theta}=1$. We now prove that $L$ satisfies (H3) with $m=2$. Let $E$ and $F$ denote measurable subsets of $M$. Since $L$ is nonnegative and self-adjoint, the Davies-Gaffney estimate (5.1) is equivalent to the property that the cosine group $\cos (t \sqrt{L}):=\frac{1}{2}\left(e^{i t \sqrt{L}}+e^{-i t \sqrt{L}}\right)$ has finite propagation speed (see Theorem 2 in [30], and Theorem 3.4 in [17]), where $\left(e^{i t \sqrt{L}}\right)_{t \in \mathbb{R}}$ is the $C_{0}$-group generated by the skewadjoint operator $i \sqrt{L}$. Therefore, there exists $c_{L}>0$ such that $\mathbf{1}_{E} \cos (t \sqrt{L}) \mathbf{1}_{F}=0$ whenever $\rho(E, F)>c_{L}|t|$. For all $z \in \mathbb{C}$ with $\operatorname{Im}( \pm z)>0$, we use the integral representation $(z I-L)^{-1}=\frac{ \pm 1}{i \sqrt{z}} \int_{0}^{\infty} e^{ \pm i \sqrt{z} t} \cos (t \sqrt{L}) d t$ (see Example 3.14.15 in [3]) to obtain

$$
\begin{aligned}
\left\|\mathbf{1}_{E}(z I-L)^{-1} \mathbf{1}_{F}\right\| & \leq \frac{1}{|z|^{1 / 2}} \int_{\rho(E, F) / c_{L}}^{\infty}\left|e^{ \pm i \sqrt{z} t}\right|\left\|\mathbf{1}_{E} \cos (t \sqrt{L}) \mathbf{1}_{F}\right\| d t \\
& \leq \frac{1}{|z|^{1 / 2}} \int_{\rho(E, F) / c_{L}}^{\infty} e^{-(\operatorname{Im}( \pm \sqrt{z})) t} d t .
\end{aligned}
$$

It is understood here that $\sqrt{z}=|z|^{1 / 2} e^{i \operatorname{Arg}(z) / 2}$ with $\operatorname{Arg}(z) \in(-\pi, \pi]$, so then $\operatorname{Im}(\sqrt{z})=|z|^{1 / 2} \sin (\operatorname{Arg}(z) / 2)$, and for each $\theta \in(0, \pi / 2)$, it follows that

$$
\left\|\mathbf{1}_{E}(z I-L)^{-1} \mathbf{1}_{F}\right\| \leq \frac{C_{\theta / 2}}{|z|} \exp \left(-\frac{\rho(E, F)|z|^{1 / 2}}{c_{L} C_{\theta / 2}}\right) \quad \forall z \in \mathbb{C} \backslash S_{\theta},
$$

which implies (H3) with $m=2$.

We have now shown that $L$ satisfies (H1)-(H3) with $\omega=0$ and $m=2$, and since $L$ satisfies (1.6), hypothesis $(\mathrm{H} 4)_{\Psi}$ holds with $q=1$ by (3.21). Therefore, except for the atomic characterisation, Theorems 3.10 and 3.13 complete the proof.

It thus remains to prove that $H_{L, \psi}^{1} \subseteq H_{L, \text { at }(N)}^{1}$ when $\psi \in \Psi_{\beta}\left(S_{\theta}^{o}\right)$ and $N>\kappa / 4$. Let $\tilde{\psi}(z)=z e^{-z}$ on $S_{\theta}^{o}$ and fix a nondegenerate even function $\eta$ in $\widetilde{\Psi}_{2 N}^{\delta}(\mathbb{R})$ such that

$$
\int_{0}^{\infty} \eta(t z) \tilde{\psi}\left(t^{2} z^{2}\right) \frac{d t}{t}=1 \quad \forall z \in S_{\theta / 2}^{o} .
$$

For example, choose any nondegenerate, even, real-valued function $\varphi \in C_{c}^{\infty}(\mathbb{R})$ supported on $[-\delta / 2, \delta / 2]$ and let $\eta(x)=\alpha\left|x^{N} \widehat{\varphi}(x)\right|^{2}$ for all $x \in \mathbb{R}$, where $\alpha$ is the normalizing constant defined by $\alpha \int_{0}^{\infty} t^{2 N} \widehat{\varphi}(t)^{2} t^{2} e^{-t^{2}} \frac{d t}{t}=1$.

Applying Proposition 4.2 with $D=\sqrt{L}$, we obtain

$$
\mathcal{S}_{\eta}^{\sqrt{L}} \mathcal{Q}_{\tilde{\psi}}^{L} u:=\int_{0}^{\infty} \eta(t \sqrt{L}) \tilde{\psi}\left(t^{2} L\right) u \frac{d t}{t}=u \quad \forall u \in \overline{\mathrm{R}(L)} .
$$

The operator $\mathcal{Q}_{\tilde{\psi}}^{L}$ has an extension $\mathcal{Q}_{\tilde{\psi}}^{L} \in \mathcal{L}\left(H_{L, \psi}^{1}, T^{1}\right)$ by $(3.16)$, since we have already established the embedding $H_{L, \psi}^{1} \subseteq L^{1}$ and that $H_{L, \psi}^{1} \cap L^{2}=E_{D, \psi}^{1}$ by Theorem 3.10. It is also the case that $\mathcal{S}_{\eta}^{\sqrt{L}}$ has an extension $\mathcal{S}_{\eta}^{\sqrt{L}} \in \mathcal{L}\left(T^{1}, H_{L, \psi}^{1}\right)$, but to prove this we must modify the theory in Section 4 to incorporate the finite 
propagation of the cosine group $\cos (t \sqrt{L})$. To this end, the fact that $\eta$ is an even function allows us to write

$$
\eta_{t}(\sqrt{L}) u=\frac{1}{\pi} \int_{0}^{\infty} \widehat{\eta}_{t}(s) \cos (s \sqrt{L}) u d s \quad \forall t>0, \forall u \in L^{2}(\mathcal{V}) .
$$

We then follow the proof of Lemma 4.4, but instead use the finite propagation of the cosine group, to deduce that

$$
\left\|\mathbf{1}_{E} \eta_{t}(\sqrt{L}) \mathbf{1}_{F}\right\| \leq \frac{1}{\pi}\|\widehat{\eta}\|_{\infty} \max \left\{\delta-\frac{\rho(E, F)}{c_{L} t}, 0\right\} \quad \forall t>0, \forall E, F \subseteq M .
$$

The extension $\mathcal{S}_{\eta}^{\sqrt{L}} \in \mathcal{L}\left(T^{1}, H_{L, \psi}^{1}\right)$ is then obtained as in Propositions 4.5 and 4.6.

Now let $u \in H_{L, \psi}^{1}$. It follows from above that $u=\mathcal{S}_{\eta}^{\sqrt{L}} U$, where $U:=\mathcal{Q}_{\tilde{\psi}}^{L} u \in T^{1}$. Therefore, in order to show that $u \in H_{L, \text { at }(N)}^{1}$, it suffices to show that $\mathcal{S}_{\eta}^{\sqrt{L}} A$ is an $H_{L}^{1}$-atom of type $N$ whenever $A$ is a $T^{1}$-atom (see the reasoning in the proof of the atomic characterisation in Theorem 4.9). To do this, note that when $A$ is supported in the tent $T(B)$ over a ball $B \subseteq M$, then (5.2) implies that $\eta(t \sqrt{L}) A_{t}$ is supported in $\alpha B$ for all $t>0$, where $\alpha>0$ only depends on $\eta$ and $L$. Following the proof of Theorem 4.9, we write $a:=\mathcal{S}_{\eta}^{\sqrt{L}} A=(\sqrt{L})^{2 N}\left(\int_{0}^{\infty} t^{2 N} \tilde{\eta}(t \sqrt{L}) A_{t} \frac{d t}{t}\right)=: L^{N} b$ for a suitable $\tilde{\eta} \in \widetilde{\Theta}(\mathbb{R})$, and then verify that $a$ and $b$ satisfy the atomic bounds in Definition 3.8. This proves that $H_{L, \psi}^{1} \subseteq H_{L, \text { at }(N)}^{1}$, which completes the proof.

We conclude by presenting a new proof of the results in Theorem 1.2 that does not rely explicitly on the ultracontractivity estimate (1.4) but instead requires that $A$ is self-adjoint with smooth coefficients.

Proof of Theorem 1.2 when $A$ is self-adjoint with smooth coefficients. Let $M=\mathbb{R}^{n}$ and consider $L=-\operatorname{div} A \nabla$ on $L^{2}(\mathcal{V})=L^{2}\left(\mathbb{R}^{n}\right)$, where $A \in L^{\infty}\left(\mathbb{R}^{n}, \mathcal{L}\left(\mathbb{C}^{n}\right)\right)$ has $C^{\infty}\left(\mathbb{R}^{n}\right)$ coefficients and is elliptic in the sense that there exists $\lambda>0$ such that

$$
\langle A(x) \zeta, \zeta\rangle_{\mathbb{C}^{n}} \geq \lambda|\zeta|^{2} \quad \forall \zeta \in \mathbb{C}^{n}, \forall x \in \mathbb{R}^{n} .
$$

This ellipticity condition, which is stronger than (1.3), implies that the matrix $A(x)$ is strictly positive and Hermitian. We proceed by introducing a first-order system $D$, a multiplication operator $B$, and a vector bundle $\mathcal{V}_{B}$, such that $L$ is a component of $(B D)^{2}$, and $B D$ satisfies the hypotheses of Theorem 4.11 on $L^{2}\left(\mathcal{V}_{B}\right)$.

Let $D_{c}: C_{c}^{\infty}\left(\mathbb{R}^{n}, \mathbb{C}^{1+n}\right) \rightarrow C_{c}^{\infty}\left(\mathbb{R}^{n}, \mathbb{C}^{1+n}\right)$ denote the symmetric, smooth-coefficient, first-order, differential operator on $L^{2}\left(\mathbb{R}^{n}, \mathbb{C}^{1+n}\right)$ defined by

$$
D_{c}=\left[\begin{array}{cc}
0 & -\operatorname{div} \\
\nabla & 0
\end{array}\right]: \begin{gathered}
C_{c}^{\infty}\left(\mathbb{R}^{n}\right) \\
C_{c}^{\infty}\left(\mathbb{R}^{n}, \mathbb{C}^{n}\right)
\end{gathered} \rightarrow \begin{gathered}
C_{c}^{\infty}\left(\mathbb{R}^{n}\right) \\
C_{c}^{\infty}\left(\mathbb{R}^{n}, \mathbb{C}^{n}\right)
\end{gathered},
$$

where $\nabla f=\left(\partial_{1} f, \ldots, \partial_{n} f\right)$ and $\operatorname{div}\left(u_{1}, \ldots, u_{n}\right)=\sum_{j=1}^{n} \partial_{j} u_{j}$. The principal symbol

$$
\sigma_{D_{c}}(x, \xi)=\left[\begin{array}{cc}
0 & -\xi^{T} \\
\xi & 0
\end{array}\right] \quad \forall x \in \mathbb{R}^{n}, \forall \xi \in \mathbb{C}^{n}
$$


satisfies (4.11), so the unique self-adjoint extension of $D_{c}$ is the operator

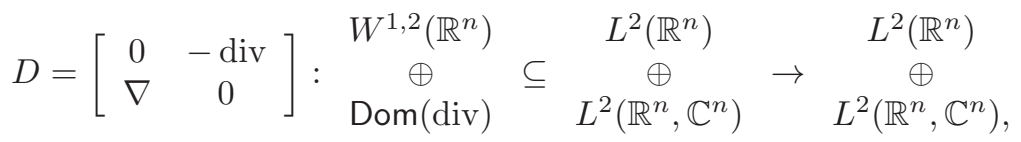

where $\nabla$ denotes the gradient extended to $W^{1,2}\left(\mathbb{R}^{n}\right)$ and div $:=-\nabla^{*}$.

Let $B(x)=\left[\begin{array}{cc}1 & 0 \\ 0 & A(x)\end{array}\right]$, so $B \in L^{\infty}\left(\mathbb{R}^{n}, \mathcal{L}\left(\mathbb{C}^{1+n}\right)\right) \cap C^{\infty}\left(\mathbb{R}^{n}, \mathcal{L}\left(\mathbb{C}^{1+n}\right)\right)$ and

$$
B D=\left[\begin{array}{cc}
0 & -\operatorname{div} \\
A \nabla & 0
\end{array}\right] \text { and }(B D)^{2}=\left[\begin{array}{cc}
L & 0 \\
0 & \tilde{L}
\end{array}\right]
$$

where $\tilde{L}:=-A \nabla$ div.

Let $\mathcal{V}_{B}$ denote the trivial bundle over $\mathbb{R}^{n}$ that has $\mathbb{C}^{1+n}$-valued sections and the smooth Hermitian metric $\langle\xi, \zeta\rangle_{\left(\mathcal{V}_{B}\right)_{x}}:=\left\langle B(x)^{-1} \xi, \zeta\right\rangle_{\mathbb{C}^{1+n}}$ for $x \in \mathbb{R}^{n}$ and $\xi, \zeta \in \mathbb{C}^{1+n}$ (since $B(x)$ is strictly positive and Hermitian, $B(x)^{-1}$ and $B(x)^{-1 / 2}$ are Hermitian; also $\left.B^{-1}, B^{-1 / 2} \in L^{\infty}\left(\mathbb{R}^{n}, \mathcal{L}\left(\mathbb{C}^{1+n}\right)\right) \cap C^{\infty}\left(\mathbb{R}^{n}, \mathcal{L}\left(\mathbb{C}^{1+n}\right)\right)\right)$. For $p \in[1,2]$, the space $L^{p}\left(\mathcal{V}_{B}\right)$ is then the set $L^{p}\left(\mathbb{R}^{n}, \mathbb{C}^{1+n}\right)$ together with the norm

$$
\|u\|_{L^{p}\left(\mathcal{V}_{B}\right)}:=\left(\int_{\mathbb{R}^{n}}\left|B(x)^{-1 / 2} u(x)\right|_{\mathbb{C}^{1+n}}^{p} d x\right)^{1 / p} \approx\|u\|_{L^{p}\left(\mathbb{R}^{n}, \mathbb{C}^{1+n}\right)}
$$

for all $u \in L^{p}\left(\mathbb{R}^{n}, \mathbb{C}^{1+n}\right)$.

We now verify the hypotheses of Theorem 4.11 for the system $B D_{c}$ on $L^{2}\left(\mathcal{V}_{B}\right)$. The inner product on $L^{2}\left(\mathcal{V}_{B}\right)$ is given by $\left\langle B^{-1} u, v\right\rangle_{L^{2}\left(\mathbb{R}^{n}, \mathbb{C}^{1+n}\right)}$, so $B D_{c}$ is symmetric on $L^{2}\left(\mathcal{V}_{B}\right)$. The principal symbol satisfies $\sigma_{B D_{c}}(x, \xi)=B(x) \sigma_{D_{c}}(x, \xi)$ and

$$
\begin{aligned}
\left|\sigma_{B D_{c}}(x, \xi) \zeta\right|_{\left(\mathcal{V}_{B}\right)_{x}} & =\left|B(x)^{1 / 2} \sigma_{D_{c}}(x, \xi) \zeta\right|_{\mathbb{C}^{n}} \\
& \leq\|B\|_{\infty}^{1 / 2}|\xi|_{\mathbb{C}^{n}}|\zeta|_{\mathbb{C}^{1+n}} \leq\|B\|_{\infty}|\xi|_{\mathbb{C}^{n}}|\zeta|_{\left(\mathcal{V}_{B}\right)_{x}}
\end{aligned}
$$

for all $x \in \mathbb{R}^{n}, \xi \in \mathbb{C}^{n}$ and $\zeta \in \mathbb{C}^{1+n}$, so $B D_{c}$ satisfies (4.11) on $\mathcal{V}_{B}$, as required.

We can now apply Theorem 4.11. In particular, consider $\underset{\sim}{p} \in[1,2], \theta \in(0, \pi / 2)$ and $\beta>n / 4$. Fix a nondegenerate $\psi \in \Psi_{\beta}\left(S_{\theta}^{o}\right)$, and let $\tilde{\psi}(z)=\psi\left(z^{2}\right)$ on $S_{\theta / 2}^{o}$ (thus $\tilde{\psi} \in \Psi_{2 \beta}\left(S_{\theta / 2}^{o}\right)$ and $\left.2 \beta>n / 2\right)$. The completion $H_{B D, \tilde{\psi}}^{p}\left(\mathcal{V}_{B}\right)$ of $E_{B D, \tilde{\psi}}^{p}\left(\mathcal{V}_{B}\right)$ in $L^{p}\left(\mathcal{V}_{B}\right)$ then exists (and $\left.H_{B D, \tilde{\psi}}^{p}\left(\mathcal{V}_{B}\right) \cap L^{2}\left(\mathcal{V}_{B}\right)=E_{B D, \tilde{\psi}}^{p}\left(\mathcal{V}_{B}\right)\right)$ by Theorem 4.11.

We now use the fact that $L$ is a component of $(B D)^{2}$ to complete the proof. Note that $L$ satisfies (H1)-(H3) with $m=2$ (see Section 3.2 ), so $E_{L, \psi}^{p}\left(\mathbb{R}^{n}\right)$ is defined with $m=2$, whereas $E_{B D, \tilde{\psi}}^{p}\left(\mathcal{V}_{B}\right)$ is defined with $m=1$ (see Lemma 4.1). Let $\varphi(z)=z e^{-z}$ on $S_{\theta}^{o}$, and let $\tilde{\varphi}(z)=\varphi\left(z^{2}\right)$ on $S_{\theta / 2}^{o}$. We use (5.3) to write

$$
\tilde{\varphi}(t B D)=t^{2}(B D)^{2} e^{-t^{2}(B D)^{2}}=\left[\begin{array}{cc}
t^{2} L e^{-t^{2} L} & 0 \\
0 & t^{2} \tilde{L} e^{-t^{2} \tilde{L}}
\end{array}\right]=\left[\begin{array}{cc}
\varphi\left(t^{2} L\right) & 0 \\
0 & \varphi\left(t^{2} \tilde{L}\right)
\end{array}\right]
$$


and then apply (3.15) to obtain

$$
\|u\|_{E_{L, \psi}^{p}\left(\mathbb{R}^{n}\right)} \approx\left\|\varphi\left(t^{2} L\right) u\right\|_{T^{p}\left(\mathbb{R}_{+}^{n+1}\right)} \approx\left\|\tilde{\varphi}(t B D)\left[\begin{array}{l}
u \\
0
\end{array}\right]\right\|_{T^{p}\left(\left(\mathcal{V}_{B}\right)_{+}\right)} \approx\left\|\left[\begin{array}{l}
u \\
0
\end{array}\right]\right\|_{E_{B D, \tilde{\psi}}^{p}\left(\mathcal{V}_{B}\right)}
$$

for all $u \in E_{L, \psi}^{p}\left(\mathbb{R}^{n}\right)$. The equivalence $L^{p}\left(\mathcal{V}_{B}\right) \approx L^{p}\left(\mathbb{R}^{n}\right)$ and the results above for $H_{B D, \tilde{\psi}}^{p}\left(\mathcal{V}_{B}\right)$ then imply that the completion $H_{L, \psi}^{p}\left(\mathbb{R}^{n}\right)$ of $E_{L, \psi}^{p}\left(\mathbb{R}^{n}\right)$ in $L^{p}\left(\mathbb{R}^{n}\right)$ exists (and $\left.H_{L, \psi}^{p}\left(\mathbb{R}^{n}\right) \cap L^{2}\left(\mathbb{R}^{n}\right)=E_{L, \psi}^{p}\left(\mathbb{R}^{n}\right)\right)$. Theorem 3.13 then provides the molecular characterisation of $H_{L, \psi}^{1}\left(\mathbb{R}^{n}\right)$. Moreover, if $N \in \mathbb{N}$ and $N>n / 4$, then $H_{B D, \tilde{\psi}}^{1}\left(\mathcal{V}_{B}\right)=H_{B D, \text { at }(2 N)}^{1}\left(\mathcal{V}_{B}\right)$ by Theorem 4.9 , which implies that $H_{L, \psi}^{1}\left(\mathbb{R}^{n}\right)$ $=H_{L, \text { at }(N)}^{1}\left(\mathbb{R}^{n}\right)$, since when $(a, \tilde{a})=(B D)^{2 N}(b, \tilde{b})$ in $L^{2}\left(\mathbb{R}^{n}\right) \oplus L^{2}\left(\mathbb{R}^{n}, \mathbb{C}^{n}\right)$ is an $H_{B D}^{1}\left(\mathcal{V}_{B}\right)$-atom of type $2 N$, then $a=L^{N} b$ is an $H_{L}^{1}\left(\mathbb{R}^{n}\right)$-atom of type $N$ by (5.3). This completes the proof.

\section{Appendix: off-diagonal estimates}

This section contains technical estimates used to prove Propositions 4.5 and 4.6. We begin with the following lemma, which allows us to manipulate $\widetilde{\Psi}(\mathbb{R})$ class functions in a manner analogous to $\Psi\left(S_{\theta}^{o}\right)$ class functions.

Lemma 6.1. Suppose that $N \in \mathbb{N}$. The following hold.

(1) For $n \in \mathbb{N}$ and $\varphi \in \widetilde{\Theta}(\mathbb{R})$, the function $\tilde{\varphi}(x):=x^{n} \varphi(x)$ for all $x \in \mathbb{R}$, is in $\widetilde{\Psi}_{n}(\mathbb{R})$. Moreover, if $\varphi \in \widetilde{\Psi}_{N}(\mathbb{R})$, then $\tilde{\varphi} \in \widetilde{\Psi}_{N+n}(\mathbb{R})$.

(2) For $m \in\{1, \ldots, N\}$ and $\eta \in \widetilde{\Psi}_{N}(\mathbb{R})$, the function $\tilde{\eta}(x):=x^{-m} \eta(x)$ for all $x \in \mathbb{R} \backslash\{0\}$, with $\tilde{\eta}(0):=\lim _{x \rightarrow 0} x^{-m} \eta(x)=\partial^{m} \eta(0) / m$ !, is in $\widetilde{\Theta}(\mathbb{R})$.

Moreover, if $m \in\{1, \ldots, N-1\}$, then $\tilde{\eta} \in \widetilde{\Psi}_{N-m}(\mathbb{R})$ (and so $\tilde{\eta}(0)=0$ ).

Proof. Suppose that $n \in \mathbb{N}$ and $\varphi \in \widetilde{\Theta}(\mathbb{R})$. The function $\tilde{\varphi}$ defined in (1) belongs to $\mathcal{S}(\mathbb{R})$ because $\varphi \in \mathcal{S}(\mathbb{R})$. The Fourier transform $\widehat{\tilde{\varphi}}$ is compactly supported because $\widehat{\varphi}$ is compactly supported and $\widehat{\tilde{\varphi}}=i^{n} \partial^{n} \widehat{\varphi}$. For each $k \in \mathbb{N}$, there exist constants $c_{k, 0}, c_{k, 1}, \ldots, c_{k, k}$ such that

$$
\partial^{k} \tilde{\varphi}(x)=\sum_{j=0}^{\min \{n-1, k\}} c_{k, j} x^{n-j} \partial^{k-j} \varphi(x)+\sum_{j=n}^{k} c_{k, j} \partial^{k-j} \varphi(x), \quad \forall x \in \mathbb{R} .
$$

It follows that $\partial^{k} \tilde{\varphi}(0)=0$ for all $k \in\{0, \ldots, n-1\}$, hence $\tilde{\varphi} \in \widetilde{\Psi}_{n}(\mathbb{R})$. Moreover, if $\varphi \in \widetilde{\Psi}_{N}(\mathbb{R})$, then $\partial^{k} \tilde{\varphi}(0)=0$ for all $k \in\{0, \ldots, N+n-1\}$, hence $\tilde{\varphi} \in \widetilde{\Psi}_{N+n}(\mathbb{R})$. This proves (1).

Now suppose that $m \in\{1, \ldots, N\}$ and $\eta \in \widetilde{\Psi}_{N}(\mathbb{R})$. The function $\tilde{\eta}$ defined in (2) satisfies the requirements of a Schwartz function, except possibly in a neighbourhood of the origin, because $\eta \in \mathcal{S}(\mathbb{R})$. The Paley-Wiener theorem guarantees 
that $\eta$ has a holomorphic extension to the entire complex plane, since $\widehat{\eta}$ is compactly supported. Therefore, there exist $\epsilon>0$ and a sequence $\left(a_{j}\right)_{j \in \mathbb{N}_{0}}$ such that the power series $a_{0}+\sum_{j=1}^{\infty} a_{j} x^{j}$ converges to $\eta(x)$ for all $x \in[-\epsilon, \epsilon]$. The assumption that $\eta \in \widetilde{\Psi}_{N}(\mathbb{R})$ implies that $a_{j}=0$ for all $j \in\{0, \ldots, N-1\}$, hence $a_{N} x^{N-m}+\sum_{j=N+1}^{\infty} a_{j} x^{j-m}$ converges to $\tilde{\eta}(x)$ for all $x \in[-\epsilon, \epsilon]$, and $\tilde{\eta} \in \mathcal{S}(\mathbb{R})$. Moreover, if $m \in\{1, \ldots, N-1\}$, then this also shows that $\partial^{k} \tilde{\eta}(0)=0$ for all $k \in\{0, \ldots, N-m-1\}$. This proves (2) provided that $\widehat{\tilde{\eta}}$ is compactly supported.

To show that $\widehat{\tilde{\eta}}$ is compactly supported when $m \in\{1, \ldots, N\}$, choose $\delta>0$ such that $\widehat{\eta}$ is supported in $[-\delta, \delta]$. It is enough to show that for each $k \in\{1, \ldots, m\}$, there exist constants $c_{k, 0}, c_{k, 1}, \ldots, c_{k, k-1}$ such that

$$
\partial^{m-k} \widehat{\tilde{\eta}}(y)= \begin{cases}\sum_{j=0}^{k-1} c_{k, j} y^{k-1-j} \int_{-\delta}^{y} x^{j} \widehat{\eta}(x) d x, & \text { if }|y| \leq \delta \\ 0, & \text { if }|y|>\delta,\end{cases}
$$

since this proves that $\widehat{\tilde{\eta}}$ is compactly supported in $[-\delta, \delta]$ by setting $k=m$.

We prove (6.1) by induction. For $k=1$, since $\eta(x)=x^{m} \tilde{\eta}(x)$, we have $\widehat{\eta}=\partial^{m} \widehat{\tilde{\eta}}$, and so $\partial^{m-1} \widehat{\tilde{\eta}}(y)=\int_{-\infty}^{y} \widehat{\eta}(x) d x$. This shows that (6.1) holds for $k=1$, since $\widehat{\eta}$ is supported in $[-\delta, \delta]$ and $\int_{-\infty}^{\infty} \widehat{\eta}(x) d x=\eta(0)=0$. Next, assume that (6.1) holds for some $k=l \in\{1, \ldots, m-1\}$. Note that $\partial^{m-(l+1)} \widehat{\tilde{\eta}}(y)=\int_{-\infty}^{y} \partial^{m-l} \widehat{\tilde{\eta}}(x) d x$. When $y<-\delta$, then $\partial^{m-(l+1)} \widehat{\tilde{\eta}}(y)=0$ by (6.1). When $y \geq-\delta$, then we use $(6.1)$ to obtain

$$
\begin{aligned}
\partial^{m-(l+1)} \widehat{\tilde{\eta}}(y) & =\int_{-\delta}^{\min \{y, \delta\}}\left(\sum_{j=0}^{l-1} c_{l, j} x^{l-1-j} \int_{-\delta}^{x} w^{j} \widehat{\eta}(w) d w\right) d x \\
& =\sum_{j=0}^{l-1} c_{l, j} \int_{-\delta}^{y}\left(\int_{w}^{\min \{y, \delta\}} x^{l-1-j} d x\right) w^{j} \widehat{\eta}(w) d w \\
& =\sum_{j=0}^{l-1} \frac{c_{l, j}}{l-j}\left(\min \{y, \delta\}^{l-j} \int_{-\delta}^{y} w^{j} \widehat{\eta}(w) d w-\int_{-\delta}^{y} w^{l} \widehat{\eta}(w) d w\right) .
\end{aligned}
$$

This shows that $(6.1)$ holds for $k=l+1$, since $\widehat{\eta}$ is supported in $[-\delta, \delta]$ and $\int_{-\infty}^{\infty} w^{j} \widehat{\eta}(w) d w=\partial^{j} \eta(0)=0$ for all $j \in\{0, \ldots, N-1\}$. We then conclude that (6.1) holds for each $k \in\{1, \ldots, m\}$. This completes the proof.

We use a proof of Auscher and Martell [7], Theorem 2.3(b), to show that polynomial off-diagonal estimates are stable under composition. This allows us to combine the off-diagonal estimates for the $\Psi\left(S_{\theta}^{o}\right)$ class in (3.9) with those for the $\widetilde{\Psi}(\mathbb{R})$ class in (4.5). We use the notation $\langle\alpha\rangle=\min \{\alpha, 1\}$ and $\langle\alpha / 0\rangle=1$ when $\alpha>0$. 
Lemma 6.2. Suppose that $C, \alpha>0$. If $\left\{T_{t}\right\}_{t>0}$ and $\left\{S_{t}\right\}_{t>0}$ are collections of operators in $\mathcal{L}\left(L^{2}(\mathcal{V})\right)$ such that

$$
\left\|\mathbf{1}_{E} T_{t} \mathbf{1}_{F}\right\| \leq C\langle t / \rho(E, F)\rangle^{\alpha} \quad \text { and } \quad\left\|\mathbf{1}_{E} S_{t} \mathbf{1}_{F}\right\| \leq C\langle t / \rho(E, F)\rangle^{\alpha}
$$

for all $t>0$ and all measurable sets $E, F \subseteq M$, then there exists $\widetilde{C}>0$ such that

$$
\left\|\mathbf{1}_{E} T_{t} S_{s} \mathbf{1}_{F}\right\| \leq \widetilde{C}\langle\max \{s, t\} / \rho(E, F)\rangle^{\alpha}
$$

for all $s, t>0$ and all measurable sets $E, F \subseteq M$.

Proof. Let $E, F \subseteq M$ denote measurable sets. The measure on $M$ is Borel with respect to the metric topology, so the set $\widetilde{E}=\{x \in M: \rho(x, E) \leq \rho(E, F) / 2\}$ is closed and hence measurable. The result follows by writing

$$
\left\|\mathbf{1}_{E} T_{t} S_{s} \mathbf{1}_{F}\right\|=\left\|\mathbf{1}_{E} T_{t}\left(\mathbf{1}_{\widetilde{E}}+\mathbf{1}_{M \backslash \widetilde{E}}\right) S_{s} \mathbf{1}_{F}\right\| \leq\left\|T_{t}\right\|\left\|\mathbf{1}_{\widetilde{E}} S_{s} \mathbf{1}_{F}\right\|+\left\|\mathbf{1}_{E} T_{t} \mathbf{1}_{M \backslash \widetilde{E}}\right\|\left\|S_{s}\right\|
$$

for all $s, t>0$, since $\rho(\widetilde{E}, F) \geq \rho(E, F) / 2$ and $\rho(E, M \backslash \widetilde{E}) \geq \rho(E, F) / 2$.

The following off-diagonal estimates are used to prove Propositions 4.5 and 4.6 .

Lemma 6.3. Suppose that $D$ is a self-adjoint operator on $L^{2}(\mathcal{V})$ and the group $\left(e^{i t D}\right)_{t \in \mathbb{R}}$ has finite propagation speed. If $m, n, N \in \mathbb{N}$ and $\delta, \sigma, \tau>0$ satisfy

$$
m \leq N, \quad m<\tau, \quad n<\sigma \quad \text { and } \quad \delta \in(0, \sigma-n),
$$

then for each $\eta \in \widetilde{\Psi}_{N}(\mathbb{R})$ and $\psi \in \Psi_{\sigma}^{\tau}\left(S_{\theta}^{o}\right)$, there exists $C>0$ such that

$$
\left\|\mathbf{1}_{E}\left(\eta_{t} \psi_{s}\right)(D) \mathbf{1}_{F}\right\| \leq C \begin{cases}(s / t)^{n}\langle t / \rho(E, F)\rangle^{\sigma-n-\delta}, & \text { if } 0<s \leq t \\ (t / s)^{m}\langle s / \rho(E, F)\rangle^{\sigma+m-\delta}, & \text { if } 0<t \leq s\end{cases}
$$

for all measurable sets $E, F \subseteq M$.

Proof. Let $E, F \subseteq M$ denote measurable sets. Suppose that $0<s \leq t$ and define

$$
\tilde{\eta}(x)=x^{n} \eta(x) \forall x \in \mathbb{R}, \quad \tilde{\psi}(z)=z^{-n} \psi(z) \forall z \in S_{\theta}^{o} \quad \text { and } \quad \tilde{\psi}(0)=0 .
$$

The function $\tilde{\eta}$ is in $\widetilde{\Psi}_{N+n}(\mathbb{R})$ by Lemma 6.1 , so Lemma 4.4 implies that

$$
\left\|\mathbf{1}_{E} \tilde{\eta}_{t}(D) \mathbf{1}_{F}\right\| \lesssim e^{-\rho(E, F) / t} \lesssim\langle t / \rho(E, F)\rangle^{\sigma-n-\delta} .
$$

The function $\tilde{\psi}$ is in $\Psi_{\sigma-n}^{\tau+n}\left(S_{\theta}^{o}\right)$, so (3.9) implies that

$$
\left\|\mathbf{1}_{E} \tilde{\psi}_{s}(D) \mathbf{1}_{F}\right\| \lesssim\langle s / \rho(E, F)\rangle^{\sigma-n-\delta} .
$$

We combine (6.3) and (6.4) using Lemma 6.2 to obtain

$$
\left\|\mathbf{1}_{E} \tilde{\eta}_{t}(D) \tilde{\psi}_{s}(D) \mathbf{1}_{F}\right\| \lesssim\langle t / \rho(E, F)\rangle^{\sigma-n-\delta}
$$


when $0<s \leq t$. The $B^{\infty}(\mathbb{R})$ functional calculus is an algebra homomorphism and $\eta_{t} \psi_{s}=(s / t)^{n} \tilde{\eta}_{t} \tilde{\psi}_{s}$ on $\mathbb{R}$, where both $\tilde{\eta}_{t}$ and $\tilde{\psi}_{s}$ are in $B^{\infty}(\mathbb{R})$. Therefore, we have $\left(\eta_{t} \psi_{s}\right)(D)=(s / t)^{n} \tilde{\eta}_{t}(D) \tilde{\psi}_{s}(D)$, and so $(6.5)$ implies $(6.2)$ when $0<s \leq t$.

Now suppose that $0<t \leq s$ and define

$$
\begin{aligned}
& \tilde{\tilde{\eta}}(x)=x^{-m} \eta(x) \forall x \in \mathbb{R} \backslash\{0\}, \tilde{\tilde{\eta}}(0)=\lim _{x \rightarrow 0} x^{-m} \eta(x), \\
& \tilde{\tilde{\psi}}(z)=z^{m} \psi(z) \forall z \in S_{\theta}^{o} \cup\{0\} .
\end{aligned}
$$

The function $\tilde{\tilde{\eta}}$ is in $\widetilde{\Theta}(\mathbb{R})$ by Lemma 6.1 , since $m \leq N$ (note that $\tilde{\tilde{\eta}}(0)=\partial^{m} \eta(0) / m$ ! and so we may have $\tilde{\tilde{\eta}}(0) \neq 0$ when $m=N)$. Lemma 4.4 then implies that $\left\|\mathbf{1}_{E} \tilde{\tilde{\eta}}_{t}(D) \mathbf{1}_{F}\right\| \lesssim e^{-\rho(E, F) / t}$. The function $\tilde{\tilde{\psi}}$ is in $\Psi_{\sigma+m}^{\tau-m}\left(S_{\theta}^{o}\right)$, so (3.9) implies that $\left\|\mathbf{1}_{E} \tilde{\tilde{\psi}}_{s}(D) \mathbf{1}_{F}\right\| \lesssim\langle s / \rho(E, F)\rangle^{\sigma+m-\delta}$. We also have $\eta_{t} \psi_{s}=(t / s)^{m} \tilde{\tilde{\eta}}_{t} \tilde{\tilde{\psi}}_{s}$ on $\mathbb{R}$, so by writing $\left(\eta_{t} \psi_{s}\right)(D)=(t / s)^{m} \tilde{\tilde{\eta}}_{t}(D) \tilde{\tilde{\psi}}_{s}(D)$ and using Lemma 6.2 to combine the two preceding estimates, we obtain $(6.2)$ when $0<t \leq s$.

Acknowledgements. We thank Alex Amenta, Lashi Bandara, Charles Batty, Andrea Carbonaro, Steve Hofmann and Pierre Portal for helpful conversations that improved the paper. We also thank the anonymous referee for providing a thorough and insightful report that improved the paper significantly.

\section{References}

[1] Albrecht, D., Duong, X. And McIntosh, A.: Operator theory and harmonic analysis. In Instructional Workshop on Analysis and Geometry, Part III (Canberra, 1995), 77-136. Proc. Centre Math. Appl. Austral. Nat. Univ. 34, Austral. Nat. Univ., Canberra, 1996.

[2] Amenta, A.: Tent spaces over metric measure spaces under doubling and related assumptions. In Operator theory in harmonic and non-commutative analysis, 23rd International Workshop in Operator Theory and its Applications (Sydney, July 2012), 1-29. Operator Theory: Advances and Applications 240, Birkhäuser, Basel, 2014.

[3] Arendt, W., Batty, C. J. K., Hieber, M. and Neubrander, F.: Vector-valued Laplace transforms and Cauchy problems. Second Edition. Monographs in Mathematics 96, Birkhäuser, Basel, 2011.

[4] Auscher, P.: Regularity theorems and heat kernel for elliptic operators. J. London Math. Soc. (2) 54 (1996), no. 2, 284-296.

[5] Auscher, P.: On necessary and sufficient conditions for $L^{p}$-estimates of Riesz transforms associated to elliptic operators on $\mathbb{R}^{n}$ and related estimates. Mem. Amer. Math. Soc. 186 (2007), no. 871, 1-75.

[6] Auscher, P., Hofmann, S., Lacey, M., McIntosh, A. and Tchamitchian, P.: The solution of the Kato square root problem for second order elliptic operators on $\mathbb{R}^{n}$. Ann. of Math. (2) 156 (2002), no. 2, 633-654.

[7] Auscher, P. And Martell, J. M.: Weighted norm inequalities, off-diagonal estimates and elliptic operators. II. Off-diagonal estimates on spaces of homogeneous type. J. Evol. Equ. 7 (2007), no. 2, 265-316. 
[8] Auscher, P., McIntosh, A. And Nahmod, A.: Holomorphic functional calculi of operators, quadratic estimates and interpolation. Indiana Univ. Math. J. 46 (1997), no. 2, 375-403.

[9] Auscher, P., McIntosh, A. And Russ, E.: Hardy spaces of differential forms on Riemannian manifolds. J. Geom. Anal. 18 (2008), no. 1, 192-248.

[10] Auscher, P. And Tchamitchian, P.: Square root problem for divergence operators and related topics. Astérisque 249 (1998), 1-172.

[11] Bernal, A.: Some results on complex interpolation of $T_{q}^{p}$ spaces. In Interpolation Spaces and Related Topics (Haifa, 1990), 1-10. Israel Math. Conf. Proc. 5, Bar-Ilan Univ., Ramat Gan, 1992.

[12] Carbonaro, A., McIntosh, A. and Morris, A. J.: Local Hardy spaces of differential forms on Riemannian manifolds. J. Geom. Anal. 23 (2013), no. 1, 106-169.

[13] Chernoff, P. R.: Essential self-adjointness of powers of generators of hyperbolic equations. J. Functional Analysis 12 (1973), 401-414.

[14] Cohn, W.S. And Verbitsky, I. E.: Factorization of tent spaces and Hankel operators. J. Funct. Anal. 175 (2000), no. 2, 308-329.

[15] Coifman, R.R., Meyer, Y. and Stein, E. M.: Some new function spaces and their applications to harmonic analysis. J. Funct. Anal. 62 (1985), no. 2, 304-335.

[16] Cowling, M., Doust, I., McIntosh, A. And Yagi, A.: Banach space operators with a bounded $H^{\infty}$ functional calculus. J. Austral. Math. Soc. Ser. A 60 (1996), no. $1,51-89$.

[17] Coulhon, T. And Sikora, A.: Gaussian heat kernel upper bounds via the Phragmén-Lindelöf theorem. Proc. Lond. Math. Soc. (3) 96 (2008), no. 2, 507-544.

[18] Davies, E. B.: Heat kernels and spectral theory. Cambridge Tracts in Mathematics 92, Cambridge University Press, Cambridge, 1989.

[19] Engel, K.-J., NAgel, R.: One-parameter semigroups for linear evolution equations. Graduate Texts in Mathematics 194, Springer-Verlag, New York, 2000.

[20] Hofmann, S., Lu, G., Mitrea, D., Mitrea, M. and Yan, L.: Hardy spaces associated to non-negative self-adjoint operators satisfying Davies-Gaffney estimates. Mem. Amer. Math. Soc. 214 (2011), no. 1007, 1-78.

[21] Hofmann, S., Mayboroda, S. and McIntosh, A.: Second order elliptic operators with complex bounded measurable coefficients in $L^{p}$, Sobolev and Hardy spaces. Ann. Sci. Éc. Norm. Supér. (4) 44 (2011), no. 5, 723-800.

[22] Harboure, E., Torrea, J. L. and Viviani, B. E.: A vector-valued approach to tent spaces. J. Analyse Math. 56 (1991), 125-140.

[23] Hytönen, T., van Neerven, J. And Portal, P.: Conical square function estimates in UMD Banach spaces and applications to $H^{\infty}$-functional calculi. J. Anal. Math. 106 (2008), 317-351.

[24] Kato, T.: Perturbation theory for linear operators. Classics in Mathematics, Springer-Verlag, Berlin, 1995.

[25] Lang, S.: Real and functional analysis. Third Edition. Graduate Texts in Mathematics 142, Springer-Verlag, New York, 1993.

[26] McIntosh, A.: Operators which have an $H_{\infty}$ functional calculus. In Miniconference on operator theory and partial differential equations (North Ryde, 1986), 210-231. Proc. Centre Math. Anal. Austral. Nat. Univ. 14, Austral. Nat. Univ., Canberra, 1986. 
[27] McIntosh, A. And Morris, A. J.: Finite propagation speed for first order systems and Hugyens' principle for hyperbolic equations. Proc. Amer. Math. Soc. 141 (2013), no. 10, 3515-3527.

[28] Morris, A. J.: Local quadratic estimates and holomorphic functional calculi. In The AMSI-ANU workshop on spectral theory and harmonic analysis, 211-231. Proc. Centre Math. Appl. Austral. Nat. Univ. 44, Austral. Nat. Univ., Canberra, 2010.

[29] Russ, E.: The atomic decomposition for tent spaces on spaces of homogeneous type. In CMA/AMSI Research Symposium "Asymptotic Geometric Analysis, Harmonic Analysis, and Related Topics", 125-135. Proc. Centre Math. Appl. Austral. Nat. Univ. 42, Austral. Nat. Univ., Canberra, 2007.

[30] SikorA, A.: Riesz transform, Gaussian bounds and the method of wave equation. Math. Z. 247 (2004), no. 3, 643-662.

[31] Taylor, M. E.: Pseudodifferential operators. Princeton Mathematical Series 34, Princeton University Press, Princeton, NJ, 1981.

[32] Wells JR., R. O.: Differential analysis on complex manifolds. Third Edition. Graduate Texts in Mathematics 65, Springer, New York, 2008.

Received August 29, 2013.

Pascal Auscher: Université de Paris-Sud, Laboratoire de Mathématiques, UMR du CNRS 8628, 91405 Orsay Cedex, France.

E-mail: Pascal.Auscher@math.u-psud.fr

Alan McIntosh: Centre for Mathematics and its Applications, Mathematical Sciences Institute, Australian National University, Canberra, ACT 0200, Australia.

E-mail: Alan.McIntosh@anu.edu.au

Andrew J. Morris: Mathematical Institute, University of Oxford, Oxford, OX2 6GG, UK.

E-mail: Andrew.Morris@maths.ox.ac.uk

Auscher and Morris thank the Mathematical Sciences Institute at the Australian National University for support during the project. Auscher was partially supported by the ANR project "Harmonic Analysis at its Boundaries," ANR-12-BS01-0013-01. McIntosh was supported by the Australian Research Council. Morris was partially supported by the American Mathematical Society and the Simons Foundation through an AMS-Simons Travel Grant. 